\title{
KOLMOGOROV OPERATOR AND FOKKER-PLANCK EQUATION ASSOCIATED TO A STOCHASTIC BURGERS EQUATION DRIVEN BY LÉVY NOISE
}

\author{
BING HU, XIAOBIN SUN AND YINGCHAO XIE
}

\begin{abstract}
In this paper, we consider a stochastic Burgers equation driven by Lévy noise and study the transition semigroup of the solution to the initial value problem for the equation in the space of continuous functions weighted by a proper potential. We show that the infinitesimal generator is the closure of the Kolmogorov operator associated to the equation in a suitable topology. We also prove existence and uniqueness results for the associated Fokker-Planck equation.
\end{abstract}

\section{Introduction and preliminaries}

This paper is concerned with a stochastic Burgers equation driven by Lévy noise on $[0,1]$. The classical Burgers equation describes the interaction between the diffusion part and the non-linear inertial part in fluid flow. Through the celebrated Hopf-Cole transformation [15], the equation can be transformed into a linear (heat) equation so the interaction can be expressed in an explicit expression in the solution. But this method no longer works in most of the random force cases. In recent years, many people have been interested in studying Burgers turbulence in the presence of random forces. Most works are with white noise, for example, [1], [2], [3], [4], [5], [6], [7], [16], [12], [13], [14], [17], [18], [19], [21] and references therein. As far as we know, there are only a few articles dealing with the case of non-white noise, see [11], [22], [23] for the Lévy noise.

Received January 21, 2014; received in final form June 26, 2014.

Research supported by the National Natural Science Foundation of China (10971180, 11271169) and the Project Funded by the Priority Academic Program Development of Jiangsu Higher Education Institutions.

2010 Mathematics Subject Classification. Primary 34D08, 34D25. Secondary 60H20. 
The aim of the present paper is to extend the results of [18] for stochastic Burgers equation driven by Wiener noise to the equation driven by Lévy noise. That is, we will investigate the transition semigroup of the solution in the space of continuous functions weighted by a proper potential and we show that the infinitesimal generator is the closure of the Kolmogorov operator associated to the equation in a suitable topology. Moreover, we prove existence and uniqueness results for the associated Fokker-Planck equation.

The paper is organized as follows. In the rest of this section, we give preliminaries and an estimate of the solution in order to define the transition semigroup and the infinitesimal generator. In the next section, we sketch the proof of the estimate of the solution. In Section 3, we study properties of the transition semigroup in $C_{b, V}\left(L^{6}\right)$. In Section 4, we study an approximate equation and prove a-priori estimates for the derivative of the transition semigroup in the space of continuous functions. Section 5, the final section, is devoted to the proof of the main results. Due to the appearance of Lévy noise, the methods for the equation driven only by Gaussian noise no longer work. We will use the techniques for studying linear SPDE and stochastic Navier-Stokes equation driven by Lévy noise developed in [8], [9], [10], [24].

Let $(\Omega, \mathscr{F}, \mathbb{P})$ be a complete probability space equipped with a filtration $\left\{\mathscr{F}_{t}, t \geq 0\right\}$ satisfying the usual conditions of completeness and right continuity, and let $N(d t, d z)$ be a Poisson random measure with intensity measure $\lambda(d z) d t$ on $\mathbb{R}^{+} \times U$, where $\lambda(d z)$ is a $\sigma$-finite measure on a measurable space $(U, \mathcal{B}(U))$. We denote by $\tilde{N}(d t, d z)$ the compensated Poisson measure $N(d t, d z)-\lambda(d z) d t$. Suppose $W_{t}$ is a $Q$-Wiener process (the precise definition will be given later) and that $W_{t}$ and $N(d t, d z)$ are independent.

Denote by $|\cdot|_{L^{p}}$ the usual norm of the space $L^{p}(0,1), p \geq 1$, and by $|\cdot|_{\infty}$ the usual supremum norm of $L^{\infty}(0,1)$. We consider the separable Hilbert space $\mathbb{H} \hat{=} L^{2}(0,1)$ (the inner product denoted by $\left.\langle\cdot, \cdot\rangle\right)$. As usual, for $k \in \mathbb{N}, p \geq 1$, $W^{k, p}(0,1)$ is the Sobolev space of all functions in $L^{p}(0,1)$ whose derivatives of order up to $k$ belong to $L^{p}(0,1), \mathbb{H}^{k}(0,1) \hat{=} W^{k, 2}(0,1)$ and $\mathbb{H}_{0}^{1}(0,1)$ is the subspace of $\mathbb{H}^{1}(0,1)$ of all functions whose trace at 0 and 1 vanishes. We define an unbounded self-adjoint operator $A$ by

$$
A x=\Delta x=\frac{\partial^{2}}{\partial \xi^{2}} x, \quad x \in \mathscr{D}(A)=\mathbb{H}^{2}(0,1) \cap \mathbb{H}_{0}^{1}(0,1) .
$$

Note that the operator $A$ is the infinitesimal generator of a strongly continuous semigroup in $\mathbb{H}$, which we denote by $e^{t A}, t \geq 0$. Moreover, the semigroup $e^{t A}$ can be extended to $L^{p}(0,1)(p>1)$,

$$
\left|e^{t A} x\right|_{L^{p}} \leq e^{\gamma_{p} t}|x|_{L^{p}}, \quad x \in L^{p}(0,1),
$$

where $\gamma_{p}=2 p^{-1}(p-1) \pi^{2}$. Note that the usual Sobolev space $W^{k, p}(0,1)$ can be extended to $W^{s, p}(0,1)$, for $s \in \mathbb{R}$, then by [18], $e^{t A}(t \geq 0)$ have the smoothing properties: for any $s_{1}, s_{2} \in \mathbb{R}$ with $s_{1} \leq s_{2}, r \geq 1, e^{t A}: W^{s_{1}, r}(0,1) \rightarrow$ 
$W^{s_{2}, r}(0,1)$ and there exists a constant $C_{1}$ depending on $s_{1}, s_{2}, r$ such that

$$
\left|e^{t A} z\right|_{W^{s_{2}, r}(0,1)} \leq C_{1}\left(1+t^{\left(s_{1}-s_{2}\right) / 2}\right)|z|_{W^{s_{1}, r}(0,1)}, \quad z \in W^{s_{1}, r}(0,1) .
$$

For $\alpha>0,(-A)^{\alpha}$ is the power of the operator $-A$, and $|\cdot|_{\alpha}$ is the norm of $\mathscr{D}\left((-A)^{\alpha / 2}\right)$ which is equivalent to the norm of $\mathbb{H}^{\alpha}(0,1) \hat{=} W^{\alpha, 2}(0,1)$. We have $|\cdot|_{0}=|\cdot|_{\mathbb{H}}$. Then,

$$
e_{k}(\xi)=\sqrt{2} \sin (k \pi \xi), \quad \xi \in[0,1], k \in \mathbb{N}
$$

are eigenfunctions of $A$ with eigenvalue $\lambda_{k}=-k^{2} \pi^{2}$. The $Q$-Wiener process $W_{t}$ is given by

$$
W_{t}=\sum_{k=1}^{\infty} \sqrt{\alpha_{k}} \beta_{t}^{k} e_{k}, \quad t \geq 0
$$

where $\alpha_{k}$ satisfies $Q e_{k}=\alpha_{k} e_{k}$ and $\left\{\beta^{k}\right\}_{k \in N}$ is a sequence of mutually independent standard Brownian motions on the stochastic basis $\left(\Omega, \mathscr{F},\left(\mathscr{F}_{t}\right)_{t \geq 0}, \mathbb{P}\right)$.

Put

$$
b(x)=\frac{1}{2} D_{\xi}\left(x^{2}\right), \quad b^{\prime}(x, y, z)=\int_{0}^{1} x(\xi) D_{\xi} y(\xi) z(\xi) d \xi .
$$

Then for any $x, y \in \mathbb{H}_{0}^{1}(0,1)$,

$$
b^{\prime}(x, x, y)=-\frac{1}{2} b^{\prime}(x, y, x), \quad b^{\prime}(x, y, y)=0
$$

and there exists a constant $C>0$ such that

$$
\begin{aligned}
|b(x)|_{\mathbb{H}} & \leq C|x|_{\mathbb{H}^{1}}^{2}, \quad|b(x)|_{\mathbb{H}^{-1}} \leq C|x|_{\mathbb{H}}|x|_{\mathbb{H}^{1}}, \\
|b(x)-b(y)|_{\mathbb{H}} & \leq C|x-y|_{\mathbb{H}^{1}}\left(|x|_{\mathbb{H}^{1}}+|y|_{\mathbb{H}^{1}}\right)
\end{aligned}
$$

and

$$
|b(x)-b(y)|_{\mathbb{H}^{-1}} \leq \frac{C}{2}|x-y|_{\mathbb{H}}\left(|x|_{\mathbb{H}^{1}}+|y|_{\mathbb{H}^{1}}\right) .
$$

In this paper, we shall study the following stochastic Burgers equation driven by Lévy noise:

$$
\left\{\begin{array}{l}
d X_{t}=\left(A X_{t}+b\left(X_{t}\right)\right) d t+d W_{t}+\int_{U} f\left(X_{t-}, u\right) \tilde{N}(d t, d u), \\
X_{0}=x, \quad x \in \mathbb{H} .
\end{array}\right.
$$

We fix a sequence of measurable subsets $U_{m}$ of $U$ with $U_{m} \uparrow U$ and $\lambda\left(U_{m}\right)<\infty$. Suppose that $f(\cdot, \cdot): \mathbb{H} \times U \rightarrow \mathbb{H}$ is measurable and satisfies $f$ maps $L^{p}(0,1) \times U$ to $L^{p}(0,1)$ for all $p \geq 1$ and that there exists a constant $K_{k}>0$ such that, for fixed $p \geq 1$ and positive integer $k$,

$\left(\mathrm{H}_{1}\right) \int_{U}|f(0, u)|_{L^{p}}^{k} \lambda(d u)<\infty$;

$\left(\mathrm{H}_{2}\right) \int_{U}|f(x, u)-f(y, u)|_{L^{p}}^{k} \lambda(d u) \leq K_{k}|x-y|_{L^{p}}^{k}, \forall x, y \in L^{p}(0,1)$;

$\left(\mathrm{H}_{3}\right) \sup _{|x|_{L^{p}} \leq C} \int_{U_{m}^{c}}|f(x, u)|_{L^{p}}^{k} \lambda(d u) \rightarrow 0$, as $m \rightarrow \infty, \forall C>0$; 
$\left(\mathrm{H}_{4}\right) f(\cdot, \cdot)$ is second order differentiable with respect to the first variable and satisfies

$$
C_{f}:=\max \left\{C_{f}^{-1,1}, C_{f}^{-3 / 4,1}, C_{f}^{1}, C_{f}^{2}\right\}<\infty
$$

where

$$
\begin{aligned}
C_{f}^{\alpha, 1} & =\sup _{x \in \mathbb{H}} \int_{U}|D f(x, u)|_{\mathscr{L}\left(\mathbb{H}^{\alpha}, \mathbb{H}^{\alpha}\right)}^{2} \lambda(d u), \\
C_{f}^{1} & =\sup _{x \in \mathbb{H}} \int_{U}|D f(x, u)|_{\mathscr{L}(\mathbb{H}, \mathbb{H})}^{4} \lambda(d u), \\
C_{f}^{2} & =\sup _{x \in \mathbb{H}} \int_{U}\left|D^{2} f(x, u)\right|_{\mathscr{L}(\mathbb{H}, \mathscr{L}(\mathbb{H}, \mathbb{H}))}^{2} \lambda(d u)
\end{aligned}
$$

$\mathscr{L}(\mathbb{H}, \mathbb{H})$ is the space of continuous linear operators from $\mathbb{H}$ to $\mathbb{H}$.

It's obvious that $\left(\mathrm{H}_{1}\right)$ and $\left(\mathrm{H}_{2}\right)$ imply there exists a constant $C>0$ such that

$$
\int_{U}|f(x, u)|_{L^{p}}^{k} \lambda(d u) \leq C\left(1+|x|_{L^{p}}^{k}\right) .
$$

REMARK 1.1. An example of $f$ satisfying $\left(\mathrm{H}_{1}\right)-\left(\mathrm{H}_{4}\right)$ is the following. Let $\lambda(\cdot)$ be a finite measure with $\int_{U}|u|_{U}^{k} \lambda(d u)<\infty$ for any positive integer $k$. By simple computation, the conditions $\left(\mathrm{H}_{1}\right)-\left(\mathrm{H}_{4}\right)$ hold for $f(x, u)=x|u|_{U}$.

If $E$ is a topological space and $E^{\prime}$ is a Banach space with norm $|\cdot|_{E^{\prime}}$, we denote by $C_{b}\left(E, E^{\prime}\right)$ the Banach space of bounded continuous $E^{\prime}$-valued function $\varphi$ on $E$ endowed with the supremum norm $\|\varphi\|_{C_{b}\left(E, E^{\prime}\right)}:=\sup _{x \in E}|\varphi(x)|_{E^{\prime}}$. For $E^{\prime}=\mathbb{R}$, we denote $C_{b}(E) \hat{=} C_{b}(E, \mathbb{R})$. If $E=\mathbb{H}$, we simply denote by $\|\cdot\|_{0}$ the supremum norm of $C_{b}(\mathbb{H})$. We also denote by $C_{b, 1}(\mathbb{H})$ the Banach space of all continuous functions on $\mathbb{H}$ with norm

$$
\|f\|_{0,1}:=\sup _{x \in \mathbb{H}}\left|f(x)\left(1+|x|_{\mathbb{H}}\right)^{-1}\right| .
$$

The set $C_{b}^{1}(\mathbb{H})$ is the space of all $\varphi \in C_{b}(\mathbb{H})$ which are Fréchet differentiable with differential $D \varphi \in C_{b}(\mathbb{H}, \mathbb{H})$. If $E$ is a Banach space, $\mathscr{M}(E)$ denotes the space of all Borel finite measures on $E$. If $\mu \in \mathscr{M}(E)$, we denote by $|\mu|_{\mathrm{TV}}$ the total variation measure of $\mu$. We shall denote by $\mathscr{M}_{V}\left(L^{6}(0,1)\right)$ the set of all $\mu \in \mathscr{M}\left(L^{6}(0,1)\right)$ such that $\int_{L^{6}(0,1)} V(x)|\mu|_{\mathrm{TV}}(d x)<\infty$, where $V(\cdot) \geq 0$ is a function on $L^{6}(0,1)$.

Suppose that $E$ is a topological space. A sequence $\left\{\varphi_{n}\right\}_{n \in \mathbb{N}} \subset C_{b}(E)$ is said to be $\pi$-convergent to a function $\varphi \in C_{b}(E)$ if $\lim _{n \rightarrow \infty} \varphi_{n}(x)=\varphi(x)(x \in E)$ and $\sup _{n \in \mathbb{N}}\left\|\varphi_{n}\right\|_{0}<\infty$. An $m$-indexed sequence $\left\{\varphi_{n_{1}, \ldots, n_{m}}\right\}_{\left(n_{1}, \ldots, n_{m}\right) \in \mathbb{N}^{m}} \subset$ $C_{b}(E)$ is said to be $\pi$-convergent to $\varphi \in C_{b}(E)$, if for $i \in\{1,2, \ldots, m-1\}$, there exists an $i$-indexed sequence $\left\{\varphi_{n_{1}, \ldots, n_{i}}\right\}_{\left(n_{1}, \ldots, n_{i}\right) \in \mathbb{N}^{i}} \subset C_{b}(E)$ such that

$$
\lim _{n_{i+1} \rightarrow \infty} \varphi_{n_{1}, \ldots, n_{i+1}} \stackrel{\pi}{=} \varphi_{n_{1}, \ldots, n_{i}}, \quad i \in\{1,2, \ldots, m-1\}
$$


and $\lim _{n_{1} \rightarrow \infty} \varphi_{n_{1}} \stackrel{\pi}{=} \varphi$. We shall write

$$
\lim _{n_{1} \rightarrow \infty} \cdots \lim _{n_{m} \rightarrow \infty} \varphi_{n_{1}, \ldots, n_{m}} \stackrel{\pi}{=} \varphi
$$

or $\varphi_{n} \stackrel{\pi}{\rightarrow} \varphi$ as $n \rightarrow \infty$, when the sequence has one index.

Using a method similar to that used in [18], [11] and [8], one can prove that (1.3) has a unique mild solution $X \in D\left([0, \infty) ; L^{p}(0,1)\right)$, the space of all $L^{p}(0,1)$-valued càdlàg functions. In fact, for finite intensity measures we can argue as in [11], and for $\sigma$-finite intensity measures we can use the argument in [8] used for dealing with the Navier-Stokes equation. The corresponding transition semigroup $P_{t}, t \geq 0$ is given by

$$
P_{t} \varphi(x)=\mathbb{E}\left[\varphi\left(X_{t}(x)\right)\right], \quad t \geq 0, \varphi \in C_{b}(\mathbb{H}), x \in \mathbb{H} .
$$

We will prove the following.

TheOREm 1.1. Under the conditions $\left(\mathrm{H}_{1}\right)-\left(\mathrm{H}_{3}\right)$, for $p \geq 2, k \geq 1$ and $T>0$, there exists a constant $C_{p, k, T}>0$ such that

$$
\mathbb{E}\left[\sup _{t \leq T}\left|X_{t}(x)\right|_{L^{p}}^{k}\right] \leq C_{p, k, T}\left(1+|x|_{L^{p}}^{k}\right)
$$

where $C_{p, k, T}$ is of the form of $C_{1}(p, k) e^{C_{2}(p, k) T}$ for some constants $C_{1}(p, k)$, $C_{2}(p, k)>0$.

Theorem 1.1 shows that the semigroup $P_{t}$ is can be extended to spaces of real valued continuous functions with polynomial growth. In particular, for $p=6, P_{t}$ acts on the space $C_{b, V}\left(L^{6}\right)$ of all continuous functions $\varphi \in L^{6}(0,1)$ such that the function $|\varphi(x)| /(1+V(x))$ is bounded, where $V(x)=|x|_{L^{6}}^{8}|x|_{L^{4}}^{2}$ $\left(x \in L^{6}(0,1)\right)$. The space $C_{b, V}\left(L^{6}\right)$, endowed with the norm

$$
\|\varphi\|_{0, V}:=\sup _{x \in L^{6}(0,1)} \frac{|\varphi(x)|}{1+V(x)},
$$

is a Banach space.

As the case in [18], $\left(P_{t}\right)_{t \geq 0}$ is not strongly continuous on $C_{b, V}\left(L^{6}\right)$ (neither on $\left.C_{b}(\mathbb{H})\right)$. However, it is strongly continuous with respect to weaker topologies. We follow here the $\pi$-convergence approach, suggested in [18] and [20]. $P_{t}$ can be also studied in other frameworks, for instance with respect to uniform convergence on compact sets, see Corollary 3.1 below.

Now, we define the infinitesimal generator of $\left(P_{t}\right)_{t \geq 0}$ by setting

$$
\mathscr{K} \varphi(x)=\lim _{t \rightarrow 0^{+}} \frac{P_{t} \varphi(x)-\varphi(x)}{t}, \quad \varphi \in \mathscr{D}\left(\mathscr{K}, C_{b, V}\left(L^{6}\right)\right), x \in L^{6}(0,1)
$$

with $\mathscr{D}\left(\mathscr{K}, C_{b, V}\left(L^{6}\right)\right)=\left\{\varphi \in C_{b, V}\left(L^{6}\right): \exists g \in C_{b, V}\left(L^{6}\right), \lim _{t \rightarrow 0^{+}}\left(P_{t} \varphi(x)-\right.\right.$ $\left.\varphi(x)) / t=g(x), \forall x \in L^{6}, \sup _{t \in(0,1)}\left\|P_{t} \varphi-\varphi\right\|_{0, V} / t<\infty\right\}$. 
The Kolmogorov operator associated to (1.3) is formally given by

$$
\begin{aligned}
\mathscr{K}_{0} \varphi(x)= & \frac{1}{2} \operatorname{Tr}\left[D^{2} \varphi(x) Q\right]+\langle x, A D \varphi(x)\rangle-\frac{1}{2}\left\langle D_{\xi} D \varphi(x), x^{\otimes 2}\right\rangle \\
& +\int_{U}[\varphi(x+f(x, u))-\varphi(x)-\langle D \varphi(x), f(x, u)\rangle] \lambda(d u),
\end{aligned}
$$

where $\varphi: L^{6}(0,1) \rightarrow \mathbb{R}$ is a suitable function.

Let $\mathscr{E}_{A}(\mathbb{H})$ be the linear span of $\varphi_{h}(x) \hat{=} e^{i\langle h, x\rangle}, h \in \mathscr{D}(A)$. The main purpose of this paper is to show that $\left(\mathscr{K}, \mathscr{D}\left(\mathscr{K}, C_{b, V}\left(L^{6}\right)\right)\right)$ is the closure of $\mathscr{K}_{0}$ defined on $\mathscr{E}_{A}(\mathbb{H})$ with respect to $\pi$-convergence. In other words, $\mathscr{E}_{A}(\mathbb{H})$ is a core for $\left(\mathscr{K}, \mathscr{D}\left(\mathscr{K}, C_{b, V}\left(L^{6}\right)\right)\right)$ and $\mathscr{K} \varphi=\mathscr{K}_{0} \varphi, \forall \varphi \in \mathscr{E}_{A}(\mathbb{H})$.

Apart from the interest to better understand the operator $\mathscr{K}_{0}$, the main motivation is to solve the Fokker-Planck equation, for any Borel measure $\mu$ on $\mathbb{H}$,

$$
\left\{\begin{array}{l}
\frac{d}{d t} \mu_{t}=\mathscr{K}_{0}^{*} \mu_{t}, \quad t \geq 0 \\
\mu_{0}=\mu .
\end{array}\right.
$$

\section{Estimates of the solution}

We will prove Theorem 1.1 and give some estimates of the solution in this section. In this paper, for simplicity, values of the constants $C, C_{p}, C_{k}, C_{p, k}, C_{p, T}, C_{p, k, T}>0$ may be different from line to line.

For $\alpha \geq 0$, let $Z_{t}^{\alpha}=\int_{0}^{t} e^{(t-s)(A-\alpha)} d W_{s}$ be the solution of the equation

$$
d Z_{t}^{\alpha}=(A-\alpha) Z_{t}^{\alpha} d t+d W_{t}, \quad Z_{0}^{\alpha}=0 .
$$

The following result is proved in [4].

Lemma 2.1. Let $q \geq 1, \varepsilon \in(0,1 / 4), \delta \in(0,1)$. Then there exists a random variable $K_{\varepsilon, \delta, q}$ such that, for all $\alpha>0$ and $t \geq 0$,

$$
\left|Z_{t}^{\alpha}\right|_{L^{q}} \leq \alpha^{-\frac{1}{4}+\varepsilon}\left(1+t^{\delta}\right) K_{\varepsilon, \delta, q},
$$

and $\mathbb{E}\left(K_{\varepsilon, \delta, q}^{k}\right)<+\infty$ for $k \geq 1$.

We define the stochastic convolution

$$
W_{A}(t)=\int_{0}^{t} e^{(t-s) A} d W_{s}=\sum_{k=1}^{\infty} \alpha_{k}^{1 / 2} e_{k} \int_{0}^{t} e^{(t-s) A} d \beta_{s}^{k} .
$$

Let $X$ be the solution of (1.3) and $Y_{t}(x) \hat{=} X_{t}(x)-W_{A}(t)$. Then we can write (1.3) as

$$
\left\{\begin{aligned}
Y_{t}(x)= & e^{t A} x+\frac{1}{2} \int_{0}^{t} e^{(t-s) A} D_{\xi}\left(Y_{s}(x)+W_{A}(s)\right)^{2} d s \\
& +\int_{0}^{t} \int_{U} e^{(t-s) A} f\left(Y_{s-}(x)+W_{A}(s), u\right) \widetilde{N}(d s, d u), \\
Y(0, x)= & x, \quad x \in \mathbb{H} .
\end{aligned}\right.
$$

$X_{t}(x)$ is called a mild solution of (1.3) if $Y_{t}(x)=X_{t}(x)-W_{A}(t)$ satisfies (2.1) $\mathbb{P}$-a.s. 
Proof of Theorem 1.1. Put $Y_{t}^{\alpha}(x)=X_{t}(x)-Z_{t}^{\alpha}$. We have

$$
\begin{aligned}
d Y_{t}^{\alpha} & =\left(A Y_{t}^{\alpha}+b\left(Y_{t}^{\alpha}+Z_{t}^{\alpha}\right)+\alpha Z_{t}^{\alpha}\right) d t+\int_{U} f\left(Y_{t-}^{\alpha}+Z_{t}^{\alpha}, u\right) \tilde{N}(d t, d u), \\
Y_{0}^{\alpha} & =x .
\end{aligned}
$$

Put $\varphi(x) \hat{=}|x|_{L^{p}}^{k}$, then we have $D \varphi(x)(\cdot)=k|x|_{L^{p}}^{k-p}\left\langle|x|^{p-2} x, I_{\mathbb{H}}(\cdot)\right\rangle$, where $I_{\mathbb{H}}(\cdot)$ is the identity transformation on $\mathbb{H}$. By Itô's formula and integrating by parts, one has

$$
\begin{aligned}
\varphi\left(Y_{t}^{\alpha}(x)\right) \\
=|x|_{L^{p}}^{k}+\int_{0}^{t}\left\langle D \varphi\left(Y_{s-}^{\alpha}(x)\right), d Y_{s}\right\rangle+N_{t}^{\varphi} \\
=|x|_{L^{p}}^{k}+k \int_{0}^{t}\left|Y_{s}^{\alpha}\right|_{L^{p}}^{k-p}\left\langle\left|Y_{s}^{\alpha}\right|^{p-2} Y_{s}, A Y_{s}^{\alpha}+b\left(Y_{s}^{\alpha}+Z_{s}^{\alpha}\right)+\alpha Z_{s}^{\alpha}\right\rangle d s \\
\quad+k \int_{0}^{t} \int_{U}\left|Y_{s-}^{\alpha}\right|_{L^{p}}^{k-p}\left\langle\left|Y_{s-}^{\alpha}\right|^{p-2} Y_{s-}^{\alpha}, f\left(Y_{s-}^{\alpha}+Z_{s}^{\alpha}, u\right)\right\rangle \tilde{N}(d s, d u)+N_{t}^{\varphi} \\
=|x|_{L^{p}}^{k}+k \int_{0}^{t}\left|Y_{s}^{\alpha}\right|_{L^{p}}^{k-p}\left[( 1 - p ) \left(\left\langle\left|Y_{s}^{\alpha}\right|^{p-2},\left|D_{\xi} Y_{s}^{\alpha}\right|^{2}\right\rangle\right.\right. \\
\left.\quad+\left\langle\left|Y_{s}^{\alpha}\right|^{p-2} Y_{s}^{\alpha} D_{\xi} Y_{s}^{\alpha}, Z_{s}^{\alpha}\right\rangle\right) \\
\left.\quad+(1-p) / 2\left\langle\left|Y_{s}^{\alpha}\right|^{p-2} D_{\xi} Y_{s}^{\alpha},\left|Z_{s}^{\alpha}\right|^{2}\right\rangle+\alpha\left\langle\left|Y_{s}^{\alpha}\right|^{p-2} Y_{s}^{\alpha}, Z_{s}^{\alpha}\right\rangle\right] d s \\
\quad+k \int_{0}^{t} \int_{U}\left|Y_{s-}\right|_{L^{p}}^{k-p}\left\langle\left|Y_{s-}^{\alpha}\right|^{p-2} Y_{s-}^{\alpha} f\left(Y_{s-}^{\alpha}+Z_{s}^{\alpha}, u\right)\right\rangle \tilde{N}(d s, d u)+N_{t}^{\varphi},
\end{aligned}
$$

where $N_{t}^{\varphi}=\int_{0}^{t} \int_{U}\left[\varphi\left(Y_{s-}^{\alpha}+f\left(X_{s-}, u\right)\right)-\varphi\left(Y_{s-}^{\alpha}\right)-\left\langle D \varphi\left(Y_{s-}^{\alpha}\right), f\left(X_{s-}, u\right)\right\rangle\right] \times$ $N(d s, d u)$.

By $\left.\left.|| Y_{s}^{\alpha}\right|^{p / 2}\right|_{\mathbb{H}}=\left|Y_{s}^{\alpha}\right|_{L^{p}}^{p / 2}$, Poincaré inequality and the interpolatory estimate

$$
|x|_{\beta} \leq|x|_{\alpha}^{\frac{\gamma-\beta}{\gamma-\alpha}}|x|_{\gamma}^{\frac{\beta-\alpha}{\gamma-\alpha}}, \quad \alpha<\beta<\gamma
$$

one has

$$
\begin{aligned}
& \left\langle\left|Y_{s}^{\alpha}\right|^{p-2} Y_{s}^{\alpha} D_{\xi} Y_{s}^{\alpha}, Z_{s}^{\alpha}\right\rangle \\
& \leq\left.\left.\left.\left.|| Y_{s}^{\alpha}\right|^{p / 2}\right|_{L^{4}}|| Y_{s}^{\alpha}\right|^{p / 2-1} D_{\xi} Y_{s}^{\alpha}\right|_{\mathbb{H}}\left|Z_{s}^{\alpha}\right|_{L^{4}} \\
& \leq\left.\left.\left.\left. C|| Y_{s}^{\alpha}\right|^{p / 2}\right|_{\mathbb{H}} \frac{1}{4}|| Y_{s}^{\alpha}\right|^{p / 2-1} D_{\xi} Y_{s}^{\alpha}\right|_{\mathbb{H}}\left|Z_{s}^{\alpha}\right|_{L^{4}} \\
& \leq\left.\left.\left.\left.\left.\left. C|| Y_{s}^{\alpha}\right|^{p / 2}\right|_{\mathbb{H}} ^{3 / 4}|| Y_{s}^{\alpha}\right|^{p / 2}\right|_{\mathbb{H}^{1}} ^{1 / 4}|| Y_{s}^{\alpha}\right|^{p / 2-1} D_{\xi} Y_{s}^{\alpha}\right|_{\mathbb{H}}\left|Z_{s}^{\alpha}\right|_{L^{4}} \\
& \leq\left.\left. C\left|Y_{s}^{\alpha}\right|_{L^{p}}^{(3 p) / 8}|| Y_{s}^{\alpha}\right|^{p / 2-1} D_{\xi} Y_{s}^{\alpha}\right|_{\mathbb{H}} ^{5 / 4}\left|Z_{s}^{\alpha}\right|_{L^{4}} \\
& \leq\left.\left.\frac{1}{4}|| Y_{s}^{\alpha}\right|^{p / 2-1} D_{\xi} Y_{s}^{\alpha}\right|_{\mathbb{H}} ^{2}+C\left|Z_{s}^{\alpha}\right|_{L^{4}}^{8 / 3}\left|Y_{s}^{\alpha}\right|_{L^{p}}^{p} .
\end{aligned}
$$


Hölder's inequality and the Agmon estimate $|x|_{L^{\infty}} \leq|x|_{\mathbb{H}}^{1 / 2}|x|_{\mathbb{H}^{1}}^{1 / 2}$ imply

$$
\begin{aligned}
& \left\langle\left|Y_{s}^{\alpha}\right|^{p-2} D_{\xi} Y_{s}^{\alpha},\left|Z_{s}^{\alpha}\right|^{2}\right\rangle \\
& \leq\left.\left.\left.\left.|| Y_{s}^{\alpha}\right|^{p / 2-1}\right|_{L^{\infty}}|| Y_{s}^{\alpha}\right|^{p / 2-1} D_{\xi} Y_{s}^{\alpha}\right|_{\mathbb{H}}\left|Z_{s}^{\alpha}\right|_{L^{4}}^{2} \\
& \leq\left.\left.\left(\left.\left.\left.\left.|| Y_{s}^{\alpha}\right|^{p / 2}\right|_{\mathbb{H}} ^{1 / 2}|| Y_{s}^{\alpha}\right|^{p / 2}\right|_{\mathbb{H}^{1}} ^{1 / 2}\right)^{(p-2) / p}|| Y_{s}^{\alpha}\right|^{p / 2-1} D_{\xi} Y_{s}^{\alpha}\right|_{\mathbb{H}^{-}}\left|Z_{s}^{\alpha}\right|_{L^{4}}^{2} \\
& \leq\left.\left. C\left|Y_{s}^{\alpha}\right|_{L^{p}}^{(p-2) / 4}|| Y_{s}^{\alpha}\right|^{p / 2-1} D_{\xi} Y_{s}^{\alpha}\right|_{\mathbb{H}} ^{(3 p-2) /(2 p)}\left|Z_{s}^{\alpha}\right|_{L^{4}}^{2} \\
& \leq\left.\left.\frac{1}{2}|| Y_{s}^{\alpha}\right|^{p / 2-1} D_{\xi} Y_{s}^{\alpha}\right|_{\mathbb{H}} ^{2}+C_{p}\left(\left|Z_{s}^{\alpha}\right|_{L^{4}}^{8}+1\right)\left(\left|Y_{s}^{\alpha}\right|_{L^{p}}^{p}+1\right)
\end{aligned}
$$

and

$$
\alpha\left\langle\left|Y_{s}^{\alpha}\right|^{p-2} Y_{s}^{\alpha}, Z_{s}^{\alpha}\right\rangle \leq \alpha\left|Y_{s}^{\alpha}\right|_{L^{p}}^{p-1}\left|Z_{s}^{\alpha}\right|_{L^{p}} \leq C_{p}\left|Z_{s}^{\alpha}\right|_{L^{p}}\left(\left|Y_{s}^{\alpha}\right|_{L^{p}}^{p}+\alpha^{p}\right) .
$$

$(2.2)-(2.5)$ yield

$$
\begin{aligned}
(2.6) \mathbb{E} \sup _{t \leq T}\left|Y_{t}^{\alpha}\right|_{L^{p}}^{k}+\left.\left.\frac{k(p-1)}{2} \mathbb{E} \int_{0}^{T}\left|Y_{s}^{\alpha}\right|_{L^{p}}^{k-p}|| Y_{s}^{\alpha}\right|^{p / 2-1} D_{\xi} Y_{s}^{\alpha}\right|_{\mathbb{H}} ^{2} d s \\
\leq|x|_{L^{p}}^{k}+k C_{p} \mathbb{E} \int_{0}^{T}\left|Y_{s}\right|_{L^{p}}^{k-p}\left[\left(\left|Z_{s}^{\alpha}\right|_{L^{4}}^{8 / 3}+\left|Z_{s}^{\alpha}\right|_{L^{4}}^{8}+\left|Z_{s}^{\alpha}\right|_{L^{p}}+1\right)\left|Y_{s}^{\alpha}\right|_{L^{p}}^{p}\right. \\
\left.+\alpha^{p}\left|Z_{s}^{\alpha}\right|_{L^{p}}+\left|Z_{s}^{\alpha}\right|_{L^{4}}^{8}+1\right] d s \\
+\mathbb{E} \int_{0}^{T} \int_{U}\left[k\left|Y_{s}^{\alpha}\right|_{L^{p}}^{k-1}\left|f\left(X_{s}, u\right)\right|_{L^{p}}\right. \\
\left.+\left|\varphi\left(Y_{s}^{\alpha}+f\left(X_{s}, u\right)\right)-\varphi\left(Y_{s}^{\alpha}\right)\right|\right] \lambda(d u) d s .
\end{aligned}
$$

By Hölder's inequality and Young's inequality (assuming from now on for simplicity that $p \geq 4$ ), one obtains

$$
\begin{aligned}
& \mathbb{E} \int_{0}^{T} \int_{U}\left|\varphi\left(Y_{s}^{\alpha}+f\left(X_{s}, u\right)\right)-\varphi\left(Y_{s}^{\alpha}\right)\right| \lambda(d u) d s \\
& \quad=\mathbb{E} \int_{0}^{T} \int_{U}\left|\int_{0}^{1}\left\langle D \varphi\left(Y_{s}^{\alpha}+\eta f\left(X_{s}, u\right)\right), f\left(X_{s}, u\right)\right\rangle d \eta\right| \lambda(d u) d s \\
& \quad \leq k \mathbb{E} \int_{0}^{T} \int_{U}\left|\int_{0}^{1}\right| Y_{s}^{\alpha}+\left.\eta f\left(X_{s}, u\right)\right|_{L^{p}} ^{k-1}\left|f\left(X_{s}, u\right)\right|_{L^{p}} d \eta \mid \lambda(d u) d s \\
& \leq C \mathbb{E} \int_{0}^{T} \int_{U}\left[\left|Y_{s}^{\alpha}\right|_{L^{p}}^{k-1}\left|f\left(X_{s}, u\right)\right|_{L^{p}}+\left|f\left(X_{s}, u\right)\right|_{L^{p}}^{k}\right] \lambda(d u) d s \\
& \leq C_{k} \mathbb{E} \int_{0}^{T}\left[\left|Y_{s}^{\alpha}\right|_{L^{p}}^{k}+\left|Y_{s}^{\alpha}\right|_{L^{p}}^{k-1}\left|Z_{s}^{\alpha}\right|_{L^{p}}+\left|Z_{s}^{\alpha}\right|_{L^{p}}^{k}+1\right] d s .
\end{aligned}
$$


(2.6) and (2.7) yield

$$
\begin{aligned}
& \mathbb{E} \sup _{t \leq T}\left|Y_{t}^{\alpha}\right|_{L^{p}}^{k}+\left.\left.\frac{k(p-1)}{2} \mathbb{E} \int_{0}^{T}\left|Y_{s}^{\alpha}\right|_{L^{p}}^{k-p}|| Y_{s}^{\alpha}\right|^{p / 2-1} D_{\xi} Y_{s}\right|_{\mathbb{H}} ^{2} d s \\
& \leq|x|_{L^{p}}^{k}+C_{p, k} \mathbb{E} \int_{0}^{T}\left[\left|Y_{s}^{\alpha}\right|_{L^{p}}^{k}\left|Z_{s}^{\alpha}\right|_{L^{p}}^{8}+\left|Y_{s}^{\alpha}\right|_{L^{p}}^{k}+\left|Z_{s}^{\alpha}\right|_{L^{p}}^{k}\right. \\
& \left.+\left|Z_{s}^{\alpha}\right|_{L^{p}}^{8}+\alpha^{p}\left|Z_{s}^{\alpha}\right|_{L^{p}}\right] d s+C_{p, k} T .
\end{aligned}
$$

Choosing $\quad \alpha=\left[(1+T)^{\frac{1}{2}} K_{\frac{1}{4}-\frac{1}{8 \vee k}, \frac{1}{2}, p}\right]^{8 \vee k}$, Lemma 2.1 implies that $\sup _{t \leq T}\left|Z_{s}^{\alpha}\right|_{L^{p}}^{8 \vee k} \leq 1$. Hence, we have

$$
\begin{gathered}
\mathbb{E} \sup _{t \leq T}\left|Y_{t}^{\alpha}\right|_{L^{p}}^{k}+\left.\left.\frac{k(p-1)}{2} \mathbb{E} \int_{0}^{T}\left|Y_{s}^{\alpha}\right|_{L^{p}}^{k-p}|| Y_{s}^{\alpha}\right|^{p / 2-1} D_{\xi} Y_{s}^{\alpha}\right|_{\mathbb{H}} ^{2} d s \\
\leq|x|_{L^{p}}^{k}+C_{p, k} \int_{0}^{T} \mathbb{E} \sup _{s \leq t}\left|Y_{s}^{\alpha}\right|_{L^{p}}^{k} d t+C_{p, k} T \mathbb{E} \alpha^{p}+C_{p, k} T .
\end{gathered}
$$

Since $T<e^{T}$ for any $T \geq 0$, there exists $C_{p, k}>0$ such that

$$
\mathbb{E} \sup _{t \leq T}\left|Y_{t}^{\alpha}\right|_{L^{p}}^{k} \leq|x|_{L^{p}}^{k}+C_{p, k} \int_{0}^{T} \mathbb{E} \sup _{s \leq t}\left|Y_{s}^{\alpha}\right|_{L^{p}}^{k} d t+C_{p, k} e^{C_{p, k} T} .
$$

Gronwall's inequality yields

$$
\mathbb{E} \sup _{t \leq T}\left|Y_{t}^{\alpha}\right|_{L^{p}}^{k} \leq C_{p, k}\left(1+|x|_{L^{p}}^{k}\right) e^{C_{p, k} T}
$$

The proof is now complete.

Put $\theta_{T}=\sup _{t \leq T}\left|W_{A}(t)\right|_{\infty}$ for $T>0$. We have $\theta_{T}<\infty, \mathbb{P}$-a.s.

Proposition 2.1. Suppose that $\left(\mathrm{H}_{1}\right)-\left(\mathrm{H}_{3}\right)$ hold and $x \in L^{p}(0,1)$ for $p \geq 2$, we have that for $T>0$,

$$
\lim _{|h|_{L^{p} \rightarrow 0}} \mathbb{E} \sup _{t \leq T}\left|X_{t}(x)-X_{t}(x+h)\right|_{L^{p}}=0
$$

or equivalently,

$$
\lim _{|h|_{L^{p} \rightarrow 0}} \mathbb{E} \sup _{t \leq T}\left|Y_{t}(x)-Y_{t}(x+h)\right|_{L^{p}}=0 .
$$

Proof. For $n \geq 1$, put

$$
\tau_{n}=\inf \left\{t>0, \sup _{|h|_{L^{p}}<\varepsilon} \sup _{s \leq t}\left|Y_{s}(x)+Y_{s}(x+h)+2 W_{A}(s)\right|_{L^{p}} \geq n\right\} .
$$

(2.1) implies that there exists a constant $C_{p}>0$ such that

$$
\begin{aligned}
& \mathbb{E} \sup _{t \leq T \wedge \tau_{n}}\left|Y_{t}(x)-Y_{t}(x+h)\right|_{L^{p}} \\
& \quad \leq C_{p}|h|_{L^{p}}+\frac{1}{2} \mathbb{E} \sup _{t \leq T \wedge \tau_{n}} \int_{0}^{t} \mid e^{(t-s) A} D_{\xi}\left(\left(Y_{s}(x)-Y_{s}(x+h)\right)\right.
\end{aligned}
$$




$$
\begin{aligned}
& \left.\times\left(Y_{s}(x)+Y_{s}(x+h)+2 W_{A}(s)\right)\right)\left.\right|_{L^{p}} d s \\
& +C_{p} \mathbb{E} \int_{0}^{T \wedge \tau_{n}} \int_{U} \mid f\left(Y_{s-}(x)+W_{A}(s), u\right) \\
& -\left.f\left(Y_{s-}(x+h)+W_{A}(s), u\right)\right|_{L^{p}} \lambda(d u) d s .
\end{aligned}
$$

Put $\Gamma(t)=1+t^{-\frac{1}{2}-\frac{1}{2 p}}$. Sobolev's embedding theorem, Hölder's inequality and (1.2) imply

$$
\begin{aligned}
& \frac{1}{2} \mathbb{E} \sup _{t \leq} \int_{0 \wedge \tau_{n}}^{t} \mid e^{(t-s) A} D_{\xi}\left(\left(Y_{s}(x)-Y_{s}(x+h)\right)\right. \\
&\left.\quad \times\left(Y_{s}(x)+Y_{s}(x+h)+2 W_{A}(s)\right)\right)\left.\right|_{L^{p}} d s \\
& \leq C \mathbb{E} \sup _{t \leq T \wedge \tau_{n}} \int_{0}^{t} \mid e^{(t-s) A} D_{\xi}\left(\left(Y_{s}(x)-Y_{s}(x+h)\right)\right. \\
&\left.\times\left(Y_{s}(x)+Y_{s}(x+h)+2 W_{A}(s)\right)\right)\left.\right|_{W^{\frac{1}{p}, \frac{p}{2}}} d s \\
& \leq C \mathbb{E} \sup _{t \leq T \wedge \tau_{n}} \int_{0}^{t} \Gamma(t-s) \mid D_{\xi}\left(\left(Y_{s}(x)-Y_{s}(x+h)\right)\right. \\
&\left.\times\left(Y_{s}(x)+Y_{s}(x+h)+2 W_{A}(s)\right)\right)\left.\right|_{W^{-1, \frac{p}{2}}} d s \\
& \leq C \mathbb{E} \sup _{t \leq T \wedge \tau_{n}} \int_{0}^{t} \Gamma(t-s) \mid\left(Y_{s}(x)-Y_{s}(x+h)\right) \\
& \quad \times\left.\left(Y_{s}(x)+Y_{s}(x+h)+2 W_{A}(s)\right)\right|_{L^{\frac{p}{2}}} d s \\
& \leq C \mathbb{E} \sup _{t \leq T \wedge \tau_{n}}^{t} \Gamma(t-s)\left|\left(Y_{s}(x)-Y_{s}(x+h)\right)\right|_{L^{p}} \\
& \quad \times\left|\left(Y_{s}(x)+Y_{s}(x+h)+2 W_{A}(s)\right)\right|_{L^{p}} d s \\
& \leq C \mathbb{E} \int_{0}^{T} n \Gamma(T-s) \sup _{s^{\prime} \leq s \wedge \tau_{n}}\left|Y_{s^{\prime}}(x)-Y_{s^{\prime}}(x+h)\right|_{L^{p}} d s .
\end{aligned}
$$

(2.8) and (2.9) imply

$$
\begin{aligned}
\mathbb{E} \sup _{t \leq T \wedge \tau_{n}}\left|Y_{t}(x)-Y_{t}(x+h)\right|_{L^{p}} \\
\leq C_{p}|h|_{L^{p}}+n C \int_{0}^{T} \Gamma(T-s) \mathbb{E} \sup _{s^{\prime} \leq s \wedge \tau_{n}}\left|Y_{s^{\prime}}(x)-Y_{s^{\prime}}(x+h)\right|_{L^{p}} d s \\
\quad+C_{p} \mathbb{E} \int_{0}^{T} \int_{U} I_{\left\{s \leq T \wedge \tau_{n}\right\}} \mid f\left(Y_{s}(x)+W_{A}(s), u\right) \\
\quad-\left.f\left(Y_{s}(x+h)+W_{A}(s), u\right)\right|_{L^{p}} \lambda(d u) d s \\
\leq C_{p}|h|_{L^{p}}+\int_{0}^{T}\left[n C \Gamma(T-s)+C_{p} K_{1}\right] \mathbb{E} \sup _{s^{\prime} \leq s \wedge \tau_{n}}\left|Y_{s^{\prime}}(x)-Y_{s^{\prime}}(x+h)\right|_{L^{p}} d s .
\end{aligned}
$$


From Gronwall's inequality,

$$
\mathbb{E} \sup _{t \leq T \wedge \tau_{n}}\left|Y_{t}(x)-Y_{t}(x+h)\right|_{L^{p}} \leq C_{p} e^{n C\left(\frac{2 p}{p-1} T^{\frac{p-1}{2 p}}+T\right)+C_{p} K_{1} T}|h|_{L^{p}} .
$$

This yields

$$
\lim _{|h|_{L^{p}} \rightarrow 0} \mathbb{E} \sup _{t \leq T \wedge \tau_{n}}\left|Y_{t}(x)-Y_{t}(x+h)\right|_{L^{p}}=0 .
$$

This and Theorem 1.1 show that this proposition is true.

\section{The transition semigroup in $C_{b, V}\left(L^{6}\right)$}

In this section, we will study properties of semigroup $\left(P_{t}\right)_{t \geq 0}$ in the space $C_{b, V}\left(L^{6}\right)$.

THEOREM 3.1. Under the conditions of Proposition 2.1, it holds that for $\varphi \in C_{b, V}\left(L^{6}\right)$

$$
\lim _{\epsilon \rightarrow 0} \sup _{|h|_{L^{6}}<\epsilon, t \in[0, T]}\left|P_{t} \varphi(x+h)-P_{t} \varphi(x)\right|=0 .
$$

Proof. For $\varphi \in C_{b}^{1}(\mathbb{H})$, one has that, for $t \leq T$,

$$
\begin{aligned}
& \left|\mathbb{E} \varphi\left(X_{t}(x+h)\right)-\mathbb{E} \varphi\left(X_{t}(x)\right)\right| \\
& \quad \leq \mathbb{E} \sup _{t \leq T}\left|\int_{0}^{1}\left\langle D \varphi\left(\eta\left(X_{t}(x+h)+(1-\eta) X_{t}(x)\right)\right), X_{t}(x+h)-X_{t}(x)\right\rangle d \eta\right| \\
& \quad \leq \sup _{x \in \mathbb{H}}|D \varphi(x)|_{\mathbb{H}} \mathbb{E} \sup _{t \leq T}\left|X_{t}(x+h)-X_{t}(x)\right|_{L^{6}} \rightarrow 0, \quad \text { as }|h|_{L^{6}} \rightarrow 0 .
\end{aligned}
$$

The density of $C_{b}^{1}(\mathbb{H})$ in $C_{b, V}\left(L^{6}\right)$ implies that, for $\varepsilon>0$, there exists $\varphi_{\varepsilon} \in$ $C_{b}^{1}(\mathbb{H})$ such that $\left\|\varphi_{\varepsilon}-\varphi\right\|_{0, V}<\varepsilon$. Hence, we have

$$
\begin{aligned}
& \varlimsup_{|h|_{L^{6} \rightarrow 0}} \mathbb{E}_{t \leq T}\left|\varphi(X(t, x+h))-\varphi\left(X_{t}(x)\right)\right| \\
& \leq \varlimsup_{|h|_{L^{6} \rightarrow 0}}\left[\mathbb{E} \sup _{t \leq T}\left|\varphi(X(t, x+h))-\varphi_{\varepsilon}(X(t, x+h))\right|\right. \\
& \left.\quad+\mathbb{E} \sup _{t \leq T}\left|\varphi_{\varepsilon}(X(t, x+h))-\varphi_{\varepsilon}\left(X_{t}(x)\right)\right|+\mathbb{E} \sup _{t \leq T}\left|\varphi_{\varepsilon}\left(X_{t}(x)\right)-\varphi\left(X_{t}(x)\right)\right|\right] \\
& \quad \leq \varlimsup_{|h|_{L^{6} \rightarrow 0}}\left[\varepsilon \mathbb{E} \sup _{t \leq T}(1+V(X(t, x+h)))+\varepsilon \mathbb{E} \sup _{t \leq T}\left(1+V\left(X_{t}(x)\right)\right)\right] \\
& \leq\left(2+C_{T, x}\right) \varepsilon .
\end{aligned}
$$

This yields

$$
\lim _{|h|_{L^{6} \rightarrow 0}} \mathbb{E} \sup _{t \leq T}\left|\varphi(X(t, x+h))-\varphi\left(X_{t}(x)\right)\right|=0 .
$$

The proof is now complete. 
Theorem 3.1 shows that $P_{t}$ maps $C_{b, V}\left(L^{6}\right)$ into itself.

ThEOREM 3.2. (1.4) defines a semigroup $\left(P_{t}\right)_{t \geq 0}$ on $C_{b, V}\left(L^{6}\right)$ and there exist constants $c_{0} \geq 1, w_{0} \in \mathbb{R}$ and a family of probability measures $\left\{\mu_{t}(x, \cdot)\right.$, $\left.t \geq 0, x \in L^{6}(0,1)\right\} \subset \mathscr{M}_{V}\left(L^{6}(0,1)\right)$ such that

(i) $P_{t} \in \mathscr{L}\left(C_{b, V}\left(L^{6}\right)\right)$ and $\left\|P_{t}\right\|_{\mathscr{L}\left(C_{b, V}\left(L^{6}\right)\right)} \leq c_{0} e^{w_{0} t}$.

(ii) $P_{t} \varphi(x)=\int_{\mathbb{H}} \varphi(y) \mu_{t}(x, d y)$ for any $t \geq 0, \varphi \in C_{b, V}\left(L^{6}\right)$ and $x \in L^{6}(0,1)$.

(iii) For $\varphi \in C_{b, V}\left(L^{6}\right)$ and $x \in L^{6}(0,1), P_{t} \varphi(x)$ is continuous with respect to $t$.

(iv) $P_{t} P_{s}=P_{t+s}$ for any $t, s \geq 0$ and $P_{0}=I$.

(v) Suppose that $\left\{\varphi, \varphi_{n}, n \geq 1\right\} \subset C_{b, V}\left(L^{6}\right)$ satisfy $\varphi_{n} /(1+V) \stackrel{\pi}{\rightarrow} \varphi /(1+V)$. We have

$$
\lim _{n \rightarrow \infty} \frac{P_{t} \varphi_{n}}{1+V} \stackrel{\pi}{=} \frac{P_{t} \varphi}{1+V}, \quad t \geq 0
$$

Proof. (i) For any $\varphi \in C_{b, V}\left(L^{6}\right)$, we can find $\left\{\varphi_{n}\right\}_{n \in \mathbb{N}} \subset C_{b}\left(L^{6}(0,1)\right)$ such that $\left|\varphi_{n}\right| \leq|\varphi|(n \geq 1)$ and $\varphi_{n}(x) \rightarrow \varphi(x), \forall x \in L^{6}(0,1)$. Theorem 1.1 implies

$$
\begin{aligned}
\mathbb{E} V\left(X_{t}(x)\right) & \leq\left(\mathbb{E}\left|X_{t}(x)\right|_{L^{6}}^{16}\right)^{\frac{1}{2}}\left(\mathbb{E}\left|X_{t}(x)\right|_{L^{4}}^{4}\right)^{\frac{1}{2}} \\
& \leq C e^{C t}\left(1+|x|_{L^{6}}^{16}\right)^{\frac{1}{2}}\left(1+|x|_{L^{4}}^{4}\right)^{\frac{1}{2}} \\
& \leq C e^{C t}\left(1+|x|_{L^{6}}^{8}\right)\left(1+|x|_{L^{4}}^{2}\right) \\
& \leq C e^{C t}(1+V(x)) .
\end{aligned}
$$

Using the dominated convergence theorem, we can define

$$
P_{t} \varphi(x)=\lim _{n \rightarrow \infty} P_{t} \varphi_{n}(x)=\lim _{n \rightarrow \infty} \mathbb{E} \varphi_{n}\left(X_{t}(x)\right) .
$$

For any $\varphi \in C_{b, V}\left(L^{6}\right)$, Theorem 3.1 yields that the function $P_{t} \varphi(x)$ on $L^{6}(0,1)$ is continuous. By $(3.1)$ and

$$
\left|P_{t} \varphi(x)\right| \leq\|\varphi\|_{0, V} \mathbb{E}\left(1+V\left(X_{t}(x)\right)\right) \leq C e^{C t}\|\varphi\|_{0, V}(1+V(x)),
$$

we obtain that (i) holds.

(ii) For $\varphi \in C_{b, V}\left(L^{6}\right)$, we take $\left(\varphi_{n}\right)_{n \geq 1} \subset C_{b}\left(L^{6}(0,1)\right)$ with $\left|\varphi_{n}\right| \leq|\varphi|$ and

$$
\lim _{n \rightarrow \infty} \frac{\varphi_{n}}{1+V} \stackrel{\pi}{=} \frac{\varphi}{1+V} \text {. }
$$

Let $\mu_{t}(x, \cdot)$ be the image measure of $X_{t}(x)$ in $\mathbb{H}$. Then the representation (ii) holds for any $\varphi_{n}$, that is,

$$
P_{t} \varphi_{n}(x)=\int_{\mathbb{H}} \varphi_{n}(y) \mu_{t}(x, d y), \quad x \in \mathbb{H} .
$$

Theorem 1.1 yields $X_{t}(x) \in L^{6}(0,1), \mathbb{P}$-a.s. for $x \in L^{6}(0,1)$. Hence, $\mu_{t}(x, \cdot)$ can be seen as a probability measure on $L^{6}(0,1)$. The dominated convergence theorem implies the result. 
(iii) Indeed, for $\varphi \in C_{b}^{2}\left(L^{6}(0,1)\right)$ with $D \varphi \in C_{b}^{1}\left(L^{6}(0,1), \mathscr{D}(A)\right)$, by using Itô's formula and taking expectation, one has

$$
\begin{aligned}
& \mathbb{E} \varphi\left(X_{t}(x)\right) \\
& =\varphi(x)+\mathbb{E} \int_{0}^{t}\left[\left\langle A D \varphi\left(X_{s}\right), X_{s}\right\rangle+\left\langle D \varphi\left(X_{s}\right), b X_{s}\right\rangle+\frac{1}{2} \operatorname{Tr}\left[D^{2} \varphi\left(X_{s}\right) Q\right]\right] d s \\
& \quad+\mathbb{E} \int_{0}^{t} \int_{U}\left[\varphi\left(X_{s}+f\left(X_{s}, u\right)\right)-\varphi\left(X_{s}\right)-\left\langle D \varphi\left(X_{s}\right), f\left(X_{s}, u\right)\right\rangle\right] \lambda(d u) d s .
\end{aligned}
$$

It is obvious that $P_{t} \varphi(x)=\mathbb{E} \varphi\left(X_{t}(x)\right)$ is continuous with respect to $t$. Thus, for any $\varphi \in C_{b, V}\left(L^{6}\right)$, the function $P_{t} \varphi(x)$ is continuous with respect to $t$.

(iv) Take $\varphi \in C_{b, V}\left(L^{6}\right)$ and consider a sequence $\left(\varphi_{n}\right)_{n \geq 1} \subset C_{b}\left(L^{6}(0,1)\right)$ such that $(1+V)^{-1} \varphi_{n} \stackrel{\pi}{=}(1+V)^{-1} \varphi$ as $n \rightarrow \infty$. By the Markov property of the process $X_{t}(x)$, (iv) holds for all $\varphi_{n}$. Then, by (ii) we find

$$
\frac{P_{t+s} \varphi}{1+V} \stackrel{\pi}{=} \lim _{n \rightarrow \infty} \frac{P_{t+s} \varphi_{n}}{1+V}=\lim _{n \rightarrow \infty} \frac{P_{t} P_{s} \varphi_{n}}{1+V} \stackrel{\pi}{=} \frac{P_{t} P_{s} \varphi}{1+V} .
$$

(v) Since (ii) holds and $\mu_{t}(x, d y) \in \mathscr{M}_{V}\left(L^{6}(0,1)\right)$, the result follows by the dominated convergence theorem.

THEOREM 3.3. Let $X_{t}(x)$ be the mild solution of (1.3) and $\left(P_{t}\right)_{t \geq 0}$ be the associated transition semigroups on $C_{b, V}\left(L^{6}\right)$. Let $\left(\mathscr{K}, \mathscr{D}\left(\mathscr{K}, C_{b, V}\left(L^{6}\right)\right)\right)$ be the infinitesimal generator defined by (1.5). Then:

(i) For $\varphi \in \mathscr{D}\left(\mathscr{K}, C_{b, V}\left(L^{6}\right)\right), P_{t} \varphi \in \mathscr{D}\left(\mathscr{K}, C_{b, V}\left(L^{6}\right)\right)$ and $\mathscr{K} P_{t} \varphi=P_{t} \mathscr{K} \varphi$, $t \geq 0$.

(ii) For $\varphi \in \mathscr{D}\left(\mathscr{K}, C_{b, V}\left(L^{6}\right)\right)$ and $x \in L^{6}(0,1)$, the function $P_{t} \varphi(x)$ is continuously differentiable on $\mathbb{R}_{+}$and

$$
\frac{d}{d t} P_{t} \varphi(x)=P_{t} \mathscr{K} \varphi(x)
$$

(iii) For $\varphi \in C_{b, V}\left(L^{6}\right)$, the function $\int_{0}^{t} P_{s} \varphi(x) d s \in \mathscr{D}\left(\mathscr{K}, C_{b, V}\left(L^{6}\right)\right)$ and

$$
\mathscr{K}\left(\int_{0}^{t} P_{s} \varphi d s\right)=P_{t} \varphi-\varphi .
$$

(iv) For $\lambda>w_{0}, w_{0}$ is the constant in Theorem 3.2, the linear operator $\mathscr{R}(\lambda, \mathscr{K})$ on $C_{b, V}\left(L^{6}\right)$ defined by

$$
\mathscr{R}(\lambda, \mathscr{K}) f(x)=\int_{0}^{\infty} e^{-\lambda t} P_{t} f(x) d t, \quad f \in C_{b, V}\left(L^{6}\right), x \in L^{6}(0,1)
$$

satisfies $\mathscr{R}(\lambda, \mathscr{K}) \in \mathscr{L}\left(C_{b, V}\left(L^{6}\right)\right)$,

$$
\|\mathscr{R}(\lambda, \mathscr{K})\|_{\mathscr{L}\left(C_{b, V}\left(L^{6}\right)\right)} \leq \frac{c_{0}}{\lambda-w_{0}},
$$


$\mathscr{R}(\lambda, \mathscr{K}) f \in \mathscr{D}\left(\mathscr{K}, C_{b, V}\left(L^{6}\right)\right)$ for $f \in C_{b, V}\left(L^{6}\right)$ and

$$
(\lambda I-\mathscr{K}) \mathscr{R}(\lambda, \mathscr{K}) f=f .
$$

$\mathscr{R}(\lambda, \mathscr{K})$ is called the resolvent of $\mathscr{K}$ at $\lambda$.

Proof. (i) For $\varphi \in \mathscr{D}\left(\mathscr{K}, C_{b, V}\left(L^{6}\right)\right)$, then $\sup _{t \in(0,1)}\left\|P_{t} \varphi-\varphi\right\|_{0, V} / t<\infty$. By Theorem 3.2(i), we have $P_{t} \varphi \in C_{b, V}\left(L^{6}\right)$ and

$$
\sup _{h \in(0,1)}\left\|P_{h}\left(P_{t} \varphi\right)-P_{t} \varphi\right\|_{0, V} / h \leq c_{0} e^{w_{0} t} \sup _{h \in(0,1)}\left\|P_{h} \varphi-\varphi\right\|_{0, V} / h<\infty .
$$

The dominated convergence theorem yields

$$
\lim _{h \rightarrow 0^{+}} \frac{P_{h}\left(P_{t} \varphi\right)(x)-P_{t} \varphi(x)}{h}=\lim _{h \rightarrow 0^{+}} P_{t}\left(\frac{P_{h} \varphi-\varphi}{h}\right)(x)=P_{t} \mathscr{K} \varphi(x) .
$$

Thus, $P_{t} \varphi \in \mathscr{D}\left(\mathscr{K}, C_{b, V}\left(L^{6}\right)\right)$ and $\mathscr{K} P_{t} \varphi=P_{t} \mathscr{K} \varphi, t \geq 0$.

(ii) It is obvious from the proof of (i).

(iii) For $\varphi \in C_{b, V}\left(L^{6}\right)$, we first check $\int_{0}^{t} P_{s} \varphi(x) d s \in C_{b, V}\left(L^{6}\right)$. For $x \in$ $L^{6}(0,1)$, Theorem 3.2 implies

$$
\left|\int_{0}^{t} P_{s} \varphi(x) d s\right| \leq c_{0}(1+V(x))\|\varphi\|_{0, V} \int_{0}^{t} e^{w_{0} s} d s
$$

that is,

$$
\sup _{x \in L^{6}} \frac{1}{1+V(x)}\left|\int_{0}^{t} P_{s} \varphi(x) d s\right|<\infty .
$$

It's easy to prove that $x \mapsto \int_{0}^{t} P_{s} \varphi(x) d s$ is continuous by Theorem 3.1. This means $\int_{0}^{t} P_{s} \varphi(x) d s \in C_{b, V}\left(L^{6}\right)$. Next, Theorem 3.2(i) implies

$$
\begin{aligned}
& \lim _{h \rightarrow 0^{+}} \frac{P_{h} \int_{0}^{t} P_{s} \varphi(x) d s-\int_{0}^{t} P_{s} \varphi(x) d s}{h} \\
& =\lim _{h \rightarrow 0^{+}} \frac{\int_{0}^{t} P_{h+s} \varphi(x) d s-\int_{0}^{t} P_{s} \varphi(x) d s}{h} \\
& =\lim _{h \rightarrow 0^{+}} \frac{\int_{t}^{t+h} P_{s} \varphi(x) d s-\int_{0}^{h} P_{s} \varphi(x) d s}{h} \\
& =P_{t} \varphi(x)-\varphi(x)
\end{aligned}
$$

and

$$
\begin{aligned}
& \sup _{h \in(0,1)}\left\|\frac{P_{h} \int_{0}^{t} P_{s} \varphi d s-\int_{0}^{t} P_{s} \varphi d s}{h}\right\|_{0, V} \\
& =\sup _{h \in(0,1)}\left\|\frac{\int_{t}^{t+h} P_{s} \varphi d s-\int_{0}^{h} P_{s} \varphi d s}{h}\right\|_{0, V} \\
& \leq C\|\varphi\|_{0, V} .
\end{aligned}
$$

Thus, $\int_{0}^{t} P_{s} \varphi(x) d s \in \mathscr{D}\left(\mathscr{K}, C_{b, V}\left(L^{6}\right)\right)$ and $\mathscr{K}\left(\int_{0}^{t} P_{s} \varphi d s\right)=P_{t} \varphi-\varphi$. 
(iv) For $f \in C_{b, V}\left(L^{6}\right)$, we have

$$
\begin{aligned}
|\mathscr{R}(\lambda, \mathscr{K}) f(x)| & \leq(1+V(x)) \int_{0}^{\infty} e^{-\lambda t}\left\|P_{t} f\right\|_{0, V} d t \\
& \leq c_{0}(1+V(x)) \int_{0}^{\infty} e^{-\lambda t} e^{w_{0} t}\|f\|_{0, V} d t
\end{aligned}
$$

and it is easy to obtain that $x \mapsto \int_{0}^{\infty} e^{-\lambda t} P_{t} f(x) d t$ is continuous by Theorem 3.1. These yield $\mathscr{R}(\lambda, \mathscr{K}) \in \mathscr{L}\left(C_{b, V}\left(L^{6}\right)\right)$ and

$$
\|\mathscr{R}(\lambda, \mathscr{K})\|_{\mathscr{L}\left(C_{b, V}\left(L^{6}\right)\right)} \leq \frac{c_{0}}{\lambda-w_{0}} .
$$

It is easy to compute

$$
\begin{aligned}
& \frac{1}{h} \int_{0}^{\infty} e^{-\lambda t}\left[P_{h+t} f(x)-P_{t} f(x)\right] d t \\
& \quad=\frac{e^{\lambda h}}{h} \int_{0}^{\infty} e^{-\lambda t} P_{t} f(x) d t-\frac{e^{\lambda h}}{h} \int_{0}^{h} e^{-\lambda t} P_{t} f(x) d t-\frac{1}{h} \int_{0}^{\infty} e^{-\lambda t} P_{t} f(x) d t \\
& \quad=\frac{e^{\lambda h}-1}{h} \int_{0}^{\infty} e^{-\lambda t} P_{t} f(x) d t-\frac{e^{\lambda h}}{h} \int_{0}^{h} e^{-\lambda t} P_{t} f(x) d t
\end{aligned}
$$

Then, we get

$$
\begin{aligned}
\lim _{h \rightarrow 0^{+}} \frac{P_{h} \mathscr{R}(\lambda, \mathscr{K}) f(x)-\mathscr{R}(\lambda, \mathscr{K}) f(x)}{h} \\
\quad=\lim _{h \rightarrow 0^{+}} \frac{1}{h} \int_{0}^{\infty} e^{-\lambda t}\left[P_{h+t} f(x)-P_{t} f(x)\right] d t \\
=\lambda \mathscr{R}(\lambda, \mathscr{K}) f(x)-f(x) .
\end{aligned}
$$

At the same time, we have

$$
\begin{aligned}
& \sup _{h \in(0,1)}\left\|\frac{P_{h} \mathscr{R}(\lambda, \mathscr{K}) f-\mathscr{R}(\lambda, \mathscr{K}) f}{h}\right\|_{0, V} \\
& \quad=\sup _{h \in(0,1)}\left\|\frac{e^{\lambda h}-1}{h} \int_{0}^{\infty} e^{-\lambda t} P_{t} f d t-\frac{e^{\lambda h}}{h} \int_{0}^{h} e^{-\lambda t} P_{t} f d t\right\|_{0, V} \\
& \leq C\|f\|_{0, V}<\infty .
\end{aligned}
$$

Thus, $\mathscr{R}(\lambda, \mathscr{K}) f \in \mathscr{D}\left(\mathscr{K}, C_{b, V}\left(L^{6}\right)\right)$ and $(\lambda I-\mathscr{K}) \mathscr{R}(\lambda, \mathscr{K}) f=f$.

In the following, we shall show that the semigroup $\left(P_{t}\right)_{t \geq 0}$ is strongly continuous with respect to another weak convergence.

TheOREM 3.4. Suppose that the conditions $\left(\mathrm{H}_{1}\right)-\left(\mathrm{H}_{3}\right)$ hold. For $p \geq 2$ and compact set $K \subset L^{p}(0,1)$, it holds

$$
\lim _{t \rightarrow 0^{+}} \sup _{x \in K} \mathbb{E}\left|X_{t}(x)-x\right|_{L^{p}}=0 \text {. }
$$


Proof. For any fixed $T>0$, we have that, for any $x \in L^{p}(0,1)$ and $0<t<T$,

$$
\begin{aligned}
& \mathbb{E}\left|X_{t}(x)-x\right|_{L^{p}} \\
& \leq\left|e^{t A} x-x\right|_{L^{p}}+\mathbb{E}\left|W_{A}(t)\right|_{L^{p}} \\
& \quad+\frac{1}{2} \mathbb{E} \int_{0}^{t}\left|e^{(t-s) A} D_{\xi}\left(Y_{s}(x)+W_{A}(s)\right)^{2}\right|_{L^{p}} d s \\
& \quad+2 \mathbb{E} \int_{0}^{t} \int_{U}\left|e^{(t-s) A} f\left(\left(Y_{s-}(x)+W_{A}(s)\right), u\right)\right|_{L^{p}} \lambda(d u) d s .
\end{aligned}
$$

Theorem 1.1 and (1.2) yield

$$
\begin{aligned}
& \mathbb{E} \int_{0}^{t}\left|e^{(t-s) A} D_{\xi}\left(Y_{s}(x)+W_{A}(s)\right)^{2}\right|_{L^{p}} d s \\
& \quad \leq C \int_{0}^{t}\left(1+(t-s)^{-1 / 2-1 / 2 p}\right) \mathbb{E}\left|\left(X_{s}(x)\right)^{2}\right|_{L^{\frac{p}{2}}} d s \\
& \quad \leq C\left(t+\frac{2 p t^{\frac{p-1}{2 p}}}{p-1}\right) \sup _{s \leq t} \mathbb{E}\left|X_{s}(x)\right|_{L^{p}}^{2} \\
& \quad \leq C e^{C t}\left(t+2 p t^{\frac{p-1}{2 p}}\right)\left(1+|x|_{L^{p}}^{2}\right) .
\end{aligned}
$$

Also by Theorem 1.1 and (1.1), we obtain

$$
\begin{aligned}
& \mathbb{E} \int_{0}^{t} \int_{U}\left|e^{(t-s) A} f\left(X_{s}(x), u\right)\right|_{L^{p}} \lambda(d u) d s \\
& \leq C \mathbb{E} \int_{0}^{t} \int_{U}\left|f\left(X_{s}(x), u\right)\right|_{L^{p}} \lambda(d u) d s \\
& \leq C \int_{0}^{t}\left(E\left|X_{s}(x)\right|_{L^{p}}+1\right) d s \leq C t e^{C t}\left(1+|x|_{L^{p}}\right)+C t .
\end{aligned}
$$

Finally, since $\left|e^{t A} x-x\right|_{L^{p}} \rightarrow 0$ uniformly on compact subsets of $L^{p}(0,1)$ and $\mathbb{E}\left|W_{A}(t)\right|_{L^{p}} \rightarrow 0$ as $t \rightarrow 0^{+}$, we can easily get the result by (3.2)-(3.4).

Thanks to Theorem 3.4 we have the following.

Corollary 3.1. $\left(P_{t}\right)_{t \geq 0}$ is a strongly continuous semigroup in the mixed topology of $C_{b, V}\left(L^{6}\right)$. That is, for $\varphi \in C_{b, V}\left(L^{6}\right)$, a compact set $K \subset L^{6}(0,1)$ and $T>0$, we have $\sup _{t \in[0, T]}\left\|P_{t} \varphi\right\|_{0, V}<\infty$ and

$$
\lim _{t \rightarrow 0^{+}} \sup _{x \in K}\left|P_{t} \varphi(x)-\varphi(x)\right|=0 .
$$

\section{The approximate problem}

Now we first consider the semigroup

$$
R_{t} \varphi(x)=\mathbb{E}\left[\varphi\left(Z_{t}(x)\right)\right], \quad t \geq 0, \varphi \in C_{b}(\mathbb{H}), x \in \mathbb{H},
$$


where $Z_{t}(x)=e^{t A} x+W_{A}(t)$ is the mild solution of the stochastic linear equation

$$
d Z_{t}=A Z_{t} d t+d W_{t}, \quad t \geq 0, \quad Z_{0}(x)=x \in \mathbb{H} .
$$

It is well known that, for $p, k \geq 1, T>0$, there exists a constant $C_{p, k, T}>0$ such that

$$
\mathbb{E} \sup _{t \in[0, t]}\left|Z_{t}(x)\right|_{L^{p}}^{k} \leq C_{p, k, T}\left(1+|x|_{L^{p}}^{k}\right) .
$$

This implies that $\left(R_{t}\right)_{t \geq 0}$ can be extended to a semigroup in the space $C_{b, 1}(\mathbb{H})$.

Define an operator by

$$
\mathscr{L} \varphi(x)=\lim _{t \rightarrow 0^{+}} \frac{R_{t} \varphi(x)-\varphi(x)}{t}, \quad \varphi \in \mathscr{D}\left(\mathscr{L}, C_{b, 1}(\mathbb{H})\right), x \in \mathbb{H}
$$

with $\mathscr{D}\left(\mathscr{L}, C_{b, 1}(\mathbb{H})\right)=\left\{\varphi \in C_{b, 1}(\mathbb{H}): \exists g \in C_{b, 1}(\mathbb{H}), \lim _{t \rightarrow 0^{+}}\left(R_{t} \varphi(x)-\varphi(x)\right) /\right.$ $t=g(x), \forall x \in \mathbb{H}$ and $\left.\sup _{t \in(0,1)}\left\|R_{t} \varphi-\varphi\right\|_{0,1} / t<\infty\right\}$. We have

$$
R_{t} e^{i\langle\cdot h\rangle}(x)=e^{i\left\langle e^{t A} x, h\right\rangle-\frac{1}{2}\left\langle Q_{t} h, h\right\rangle}, \quad t \geq 0, x, h \in \mathbb{H}
$$

and that $\mathscr{E}_{A}(\mathbb{H})$ is stable for $\left(R_{t}\right)_{t \geq 0}$, where $Q_{t} x=\int_{0}^{t} e^{s A} Q e^{s A} x d s$.

Denote by $\mathscr{L}_{0}$ the OU operator

$$
\mathscr{L}_{0} \varphi(x)=\frac{1}{2} \operatorname{Tr}\left[D^{2} \varphi(x) Q\right]+\langle x, A D \varphi(x)\rangle, \quad \varphi \in \mathscr{E}_{A}(\mathbb{H}), x \in \mathbb{H} .
$$

For $\varphi=e^{i\langle x, h\rangle}$, it is easy to prove $\mathscr{L}_{0} \varphi \in C_{b, 1}(\mathbb{H})$.

We have $\varphi \in \mathscr{D}\left(\mathscr{L}, C_{b, 1}(\mathbb{H})\right)$ and $\mathscr{L} \varphi(x)=\mathscr{L}_{0} \varphi(x)$ for $\varphi \in \mathscr{E}_{A}(\mathbb{H})$ and $x \in \mathbb{H}$ (see [7] and [18]). This means that $\mathscr{E}_{A}(\mathbb{H})$ is a $\pi$-core for $\left(\mathscr{L}, \mathscr{D}\left(\mathscr{L}, C_{b, 1}(\mathbb{H})\right)\right)$ and, for $\varphi \in \mathscr{D}\left(\mathscr{L}, C_{b, 1}(\mathbb{H})\right)$, there exists $m \in \mathbb{N}$ and an $m$-indexed sequence $\left(\varphi_{n_{1}, \ldots, n_{m}}\right)_{n_{1}, \ldots, n_{m} \in \mathbb{N}} \subset \mathscr{E}_{A}(\mathbb{H})$ such that

$$
\begin{array}{r}
\lim _{n_{1} \rightarrow \infty} \cdots \lim _{n_{m} \rightarrow \infty} \frac{\varphi_{n_{1}, \ldots, n_{m}}}{1+|\cdot|_{\mathbb{H}}} \stackrel{\frac{\pi}{=}}{\frac{\varphi}{1+|\cdot|_{\mathbb{H}}},} \\
\lim _{n_{1} \rightarrow \infty} \cdots \lim _{n_{m} \rightarrow \infty} \frac{\mathscr{L}_{0} \varphi_{n_{1}, \ldots, n_{m}}}{1+|\cdot|_{\mathbb{H}}} \stackrel{\pi}{=} \frac{\mathscr{L} \varphi}{1+|\cdot|_{\mathbb{H}}} .
\end{array}
$$

If $\varphi \in \mathscr{D}\left(\mathscr{L}, C_{b, 1}(\mathbb{H})\right) \cap C_{b}^{1}(\mathbb{H})$, we can further get

$$
\lim _{n_{1} \rightarrow \infty} \ldots \lim _{n_{m} \rightarrow \infty}\left\langle D \varphi_{n_{1}, \ldots, n_{m}}, h\right\rangle \stackrel{\pi}{=}\langle D \varphi, h\rangle, \quad h \in \mathbb{H} .
$$

For $T>0,(4.1)$ yields that there exists $C_{T}>0$ such that

$$
\mathbb{E}\left[\sup _{t \leq T} V\left(Z_{t}(x)\right)\right] \leq C_{T}(1+V(x)) .
$$

This shows that $R_{t}$ acts on $C_{b, V}\left(L^{6}\right)$.

Put $\mathscr{D}\left(\mathscr{L}_{V}, C_{b, V}\left(L^{6}\right)\right)=\left\{\varphi \in C_{b, V}\left(L^{6}\right): \exists g \in C_{b, V}\left(L^{6}\right), \lim _{t \rightarrow 0^{+}}\left(R_{t} \varphi(x)-\right.\right.$ $\varphi(x)) / t=g(x), \forall x \in L^{6}(0,1)$ and $\left.\sup _{t \in(0,1)}\left\|R_{t} \varphi-\varphi\right\|_{0, V} / t<\infty\right\}$ and

$$
\mathscr{L}_{V} \varphi(x)=\lim _{t \rightarrow 0^{+}} \frac{R_{t} \varphi(x)-\varphi(x)}{t}, \quad \varphi \in \mathscr{D}\left(\mathscr{L}_{V}, C_{b, V}\left(L^{6}\right)\right), x \in L^{6}(0,1) .
$$


Then $\left(\mathscr{L}_{V}, \mathscr{D}\left(\mathscr{L}_{V}, C_{b, V}\left(L^{6}\right)\right)\right)$ is the infinitesimal generator of $R_{t}$ on $C_{b, V}\left(L^{6}\right)$, $\mathscr{E}_{A}(\mathbb{H}) \subset \mathscr{D}\left(\mathscr{L}_{V}, C_{b, V}\left(L^{6}\right)\right)$ and $\mathscr{L}_{V} \varphi(x)=\mathscr{L} \varphi(x)=\mathscr{L}_{0} \varphi(x)$ for $\varphi \in \mathscr{E}_{A}(\mathbb{H})$ and $x \in L^{6}(0,1)$.

In the following, we consider some approximate equations. For $m \geq 1$, denoted by $T_{m}$ the projection operator from $\mathbb{H}$ to the linear space $\mathbb{H}_{m}$ generated by $\left\{e_{i}, i=1, \ldots, m\right\}$.

For $m \in \mathbb{N}$, set $b_{m}(x)=T_{m} b\left(T_{m} x\right), x \in \mathbb{H}$ and $W_{t}^{m}=T_{m} W_{t}$. We consider the approximate equation

$$
\left\{\begin{aligned}
d X_{t}^{m}= & \left(A X_{t}^{m}+b_{m}\left(X_{t}^{m}\right)\right) d t+d W_{t}^{m} \\
& +\int_{U} T_{m} f\left(T_{m} X_{t-}^{m}, u\right) \tilde{N}(d t, d u), \\
X_{0}^{m}= & x, \quad x \in \mathbb{H}_{m},
\end{aligned}\right.
$$

whose solution is denoted by $X_{t}^{m}(x)$ and

$$
\begin{aligned}
X_{t}^{m}(x)= & e^{t A} x+\frac{1}{2} \int_{0}^{t} e^{(t-s) A} T_{m} D_{\xi}\left(T_{m} X_{s}^{m}(x)\right)^{2} d s+\int_{0}^{t} e^{(t-s) A} d W_{s}^{m} \\
& +\int_{0}^{t} \int_{U} e^{(t-s) A} T_{m} f\left(T_{m} X_{s-}^{m}(x), u\right) \widetilde{N}(d s, d u) .
\end{aligned}
$$

Put $W_{A}^{m}(t)=\int_{0}^{t} e^{(t-s) A} d W_{s}^{m}$ and $Y_{t}^{m}(x)=X_{t}^{m}(x)-W_{A}^{m}(t)$. Then we have

$$
\begin{aligned}
Y_{t}^{m}(x)= & e^{t A} x+\frac{1}{2} \int_{0}^{t} e^{(t-s) A} T_{m} D_{\xi}\left(T_{m} Y_{s}^{m}(x)+W_{A}^{m}(s)\right)^{2} d s \\
& +\int_{0}^{t} \int_{U} e^{(t-s) A} T_{m} f\left(T_{m}\left(Y_{s-}^{m}(x)+W_{A}^{m}(s), u\right)\right) \widetilde{N}(d s, d u) .
\end{aligned}
$$

Since $\left\langle b_{m}(x), x\right\rangle=\left\langle T_{m} b\left(T_{m}(x)\right), x\right\rangle=\left\langle b\left(T_{m}(x)\right), T_{m} x\right\rangle=0$, all the above theorems and propositions hold uniformly on $m$ and we have the following result.

Theorem 4.1. Suppose that $\left(\mathrm{H}_{1}\right)-\left(\mathrm{H}_{4}\right)$ hold. For $p \geq 2, x \in L^{p}(0,1)$, (4.2) has a unique solution $X^{m} \in L^{p}(0,1)$. Moreover,

$$
\lim _{m \rightarrow \infty} \mathbb{E} \sup _{t \leq T}\left|X_{t}^{m}(x)-X_{t}(x)\right|_{L^{p}}=0, \quad T>0 .
$$

Proof. We only prove that (4.3) is true. For $k \geq 1$, set

$$
\tau_{k}:=\inf \left\{t>0, \sup _{s \leq t}\left|X_{s}(x)\right|_{L^{p}} \geq k\right\} .
$$

Then $\left(\tau_{k}\right)_{k \geq 1}$ is a sequence of stopping times with $\tau_{k} \uparrow \infty$ as $k \uparrow \infty$. For $m \geq 1$, one has

$$
\begin{aligned}
& \left|Y_{t}^{m}(x)-Y_{t}(x)\right|_{L^{p}} \\
& \quad \leq \frac{1}{2} \int_{0}^{t} \mid e^{(t-s) A}\left[T_{m} D_{\xi}\left(T_{m} Y_{s}^{m}(x)+W_{A}^{m}(s)\right)^{2}\right. \\
& \left.\quad-D_{\xi}\left(Y_{s}(x)+W_{A}(s)\right)^{2}\right]\left.\right|_{L^{p}} d s
\end{aligned}
$$




$$
\begin{aligned}
& +\mid \int_{0}^{t} \int_{U} e^{(t-s) A}\left[T_{m} f\left(T_{m}\left(Y_{s-}^{m}(x)+W_{A}^{m}(s)\right), u\right)\right. \\
& \left.-f\left(Y_{s-}(x)+W_{A}(s), u\right)\right]\left.\tilde{N}(d s, d u)\right|_{L^{p}}
\end{aligned}
$$

and

$$
\begin{aligned}
& \int_{0}^{t} \mid e^{(t-s) A}\left[T_{m} D_{\xi}\left(T_{m} Y_{s}^{m}(x)+W_{A}^{m}(s)\right)^{2}\right. \\
&\left.\quad-D_{\xi}\left(Y_{s}(x)+W_{A}(s)\right)^{2}\right]\left.\right|_{L^{p}} d s \\
& \leq C \int_{0}^{t} \Gamma(t-s)\left|\left(T_{m} Y_{s}^{m}(x)+W_{A}^{m}(s)\right)^{2}-\left(Y_{s}(x)+W_{A}(s)\right)^{2}\right|_{L^{\frac{p}{2}}} d s \\
&+\int_{0}^{t} \mid e^{(t-s) A}\left[T_{m} D_{\xi}\left(Y_{s}(x)+W_{A}(s)\right)^{2}\right. \\
&\left.-D_{\xi}\left(Y_{s}(x)+W_{A}(s)\right)^{2}\right]\left.\right|_{L^{p}} d s \\
& \leq C \int_{0}^{t} \Gamma(t-s)\left(\left|T_{m} Y_{s}^{m}(x)-Y_{s}(x)\right|_{L^{p}}+\left|W_{A}^{m}(s)-W_{A}(s)\right|_{L^{p}}\right) \\
& \times\left|T_{m} Y_{s}^{m}(x)+W_{A}^{m}(s)+Y_{s}(x)+W_{A}(s)\right|_{L^{p}} d s \\
&+\int_{0}^{t} \mid e^{(t-s) A}\left[T_{m} D_{\xi}\left(Y_{s}(x)+W_{A}(s)\right)^{2}\right. \\
&\left.-D_{\xi}\left(Y_{s}(x)+W_{A}(s)\right)^{2}\right]\left.\right|_{L^{p}} d s .
\end{aligned}
$$

By Theorem 1.1, we have

$$
\begin{aligned}
\sup _{t \leq T \wedge \tau_{k}} \int_{0}^{t}\left|e^{(t-s) A} D_{\xi}\left(X_{s}(x)\right)^{2}\right|_{L^{p}} d s & \leq C \sup _{t \leq T \wedge \tau_{k}} \int_{0}^{t} \Gamma(t-s)\left|\left(X_{s}(x)\right)^{2}\right|_{L^{\frac{p}{2}}} d s \\
& \leq C_{T} \sup _{t \leq T}\left|\left(Y_{t}(x)+W_{A}(t)\right)\right|_{L^{p}}^{2}<\infty \quad \text { a.s. }
\end{aligned}
$$

This implies

$$
\begin{gathered}
\lim _{m \rightarrow \infty} \mathbb{E} \sup _{t \leq T \wedge \tau_{k}} \int_{0}^{t} \mid T_{m} e^{(t-s) A} D_{\xi}\left(Y_{s}(x)+W_{A}(s)\right)^{2} \\
-\left.e^{(t-s) A} D_{\xi}\left(Y_{s}(x)+W_{A}(s)\right)^{2}\right|_{L^{p}} d s=0
\end{gathered}
$$

and

$$
\lim _{m \rightarrow \infty} \mathbb{E} \sup _{s \leq T}\left|W_{A}^{m}(s)-W_{A}(s)\right|_{L^{p}}=0
$$


On the other hand,

$$
\begin{aligned}
& \mathbb{E} \sup _{t \leq T \wedge \tau_{k}} \mid \int_{0}^{t} \int_{U} e^{(t-s) A}\left[T_{m} f\left(T_{m}\left(Y_{s-}^{m}(x)+W_{A}^{m}(s)\right), u\right)\right. \\
&\left.\quad-f\left(Y_{s-}(x)+W_{A}(s), u\right)\right]\left.\tilde{N}(d s, d u)\right|_{L^{p}} \\
& \leq C \mathbb{E} \int_{0}^{T \wedge \tau_{k}} \int_{U} \mid T_{m}\left[f\left(T_{m}\left(Y_{s}^{m}(x)+W_{A}^{m}(s)\right), u\right)\right. \\
&\left.-f\left(Y_{s}(x)+W_{A}(s), u\right)\right]\left.\right|_{L^{p}} \lambda(d u) d s \\
&+C \mathbb{E} \int_{0}^{T} \int_{U}\left|\left(I-T_{m}\right) f\left(Y_{s}(x)+W_{A}(s), u\right)\right|_{L^{p}} \lambda(d u) d s \\
& \leq C \mathbb{E} \int_{0}^{T}\left|Y_{s \wedge \tau_{k}}^{m}(x)-Y_{s \wedge \tau_{k}}(x)\right|_{L^{p}} d s \\
&+C T \mathbb{E} \sup _{s \leq T}\left|T_{m} Y_{s}^{m}(x)-Y_{s}^{m}(x)\right|_{L^{p}} \\
&+C T \mathbb{E} \sup _{s \leq T}\left|W_{A}^{m}(s)-W_{A}(s)\right|_{L^{p}} \\
&+C \mathbb{E} \int_{0}^{T} \int_{U}\left|\left(I-T_{m}\right) f\left(Y_{s}(x)+W_{A}(s), u\right)\right|_{L^{p}} \lambda(d u) d s .
\end{aligned}
$$

The conditions $\left(\mathrm{H}_{1}\right)$ and $\left(\mathrm{H}_{2}\right)$ yield

$$
\lim _{m \rightarrow \infty} \mathbb{E} \int_{0}^{T} \int_{U}\left|\left(I-T_{m}\right) f\left(Y_{s-}(x)+W_{A}(s), u\right)\right|_{L^{p}} \lambda(d u) d s=0 .
$$

By using

$$
\begin{aligned}
& \sup _{s \leq T}\left|T_{m} Y_{s}^{m}(x)-Y_{s}^{m}(x)\right|_{L^{p}} \\
& \quad=\sup _{s \leq T}\left|e^{s A}\left(x-x^{m}\right)\right|_{L^{p}} \leq e^{\gamma_{p} T}\left|x-x^{m}\right|_{L^{p}} \rightarrow 0, \quad m \rightarrow \infty
\end{aligned}
$$

and Gronwall's inequality, (4.6)-(4.9) imply that, for $k \geq 1$,

$$
\lim _{m \rightarrow \infty} \mathbb{E} \sup _{t \leq T \wedge \tau_{k}}\left|Y_{t}^{m}(x)-Y_{t}(x)\right|_{L^{p}}=0
$$

Finally, since

$$
\begin{aligned}
& \mathbb{E} \sup _{t \leq T}\left|Y_{t}^{m}(x)-Y_{t}(x)\right|_{L^{p}} \\
& \leq \mathbb{E} \sup _{t \leq T \wedge \tau_{k}}\left|Y_{t}^{m}(x)-Y_{t}(x)\right|_{L^{p}} \\
& \quad+\left(P\left(\tau_{k}<T\right)\right)^{\frac{1}{2}} \mathbb{E}\left[\sup _{t \leq T}\left|Y_{t}^{m}(x)-Y_{t}(x)\right|_{L^{p}}^{2}\right]^{\frac{1}{2}} \\
& \quad \leq \mathbb{E} \sup _{t \leq T \wedge \tau_{k}}\left|Y_{t}^{m}(x)-Y_{t}(x)\right|_{L^{p}}
\end{aligned}
$$




$$
\begin{aligned}
& \quad+\mathbb{P}\left[\sup _{s \leq T}\left|X_{s}(x)\right|_{L^{p}} \geq k\right]^{\frac{1}{2}} \mathbb{E}\left[\sup _{t \leq T}\left|X_{t}^{m}(x)-X_{t}(x)\right|_{L^{p}}^{2}\right]^{\frac{1}{2}} \\
& \leq \mathbb{E} \sup _{t \leq T \wedge \tau_{k}}\left|Y_{t}^{m}(x)-Y_{t}(x)\right|_{L^{p}}+\frac{1}{k} \mathbb{E} \sup _{s \leq T}\left|X_{s}(x)\right|_{L^{p}}^{2} .
\end{aligned}
$$

First, let $k \rightarrow \infty$ and then let $m \rightarrow \infty$ Theorem 1.1 and (4.10) show that (4.3) is valid.

We denote by $P_{t}^{m}$ the transition semigroup

$$
P_{t}^{m} \varphi(x)=\mathbb{E}\left[\varphi\left(X_{t}^{m}(x)\right)\right], \quad t \geq 0, \varphi \in C_{b, V}\left(L^{6}\right), x \in L^{6}(0,1) .
$$

By a standard argument, we have

$$
\lim _{m \rightarrow \infty} \frac{P_{t}^{m} \varphi}{1+V} \stackrel{\pi}{=} \frac{P_{t} \varphi}{1+V}, \quad t \geq 0 .
$$

For $m \geq 1$, we can define the infinitesimal generator $\left(\mathscr{K}_{m}, \mathscr{D}\left(\mathscr{K}_{m}\right.\right.$, $\left.C_{b, V}\left(L^{6}\right)\right)$ ) of the semigroup $\left(P_{t}^{m}\right)_{t \geq 0}$ as (1.5). It is clear that all results in Section 3 hold for $\left(P_{t}^{m}\right)_{t \geq 0}$ and $\left(\mathscr{K}_{m}, \mathscr{D}\left(\mathscr{K}_{m}, C_{b, V}\left(L^{6}\right)\right)\right)$.

In the following, we shall give a-priori estimates.

As the case in [18], a-priori estimates on $D P_{t} \varphi$ for $D_{x} X_{t}(x)$ cannot be applied here. As the authors did in [18] and [20], we consider a Kolmogorov operator with an additional potential term, for $c>0$ sufficiently large,

$$
\mathscr{K}_{0}^{\prime} \varphi(x)=\mathscr{K}_{0} \varphi(x)-c|x|_{L^{4}}^{4} \varphi(x), \quad \varphi \in \mathscr{E}_{A}(\mathbb{H}) .
$$

By the Feynman-Kac formula, we have the corresponding semigroup

$$
S_{t} \varphi(x)=\mathbb{E}\left[e^{-c \int_{0}^{t}\left|X_{s}(x)\right|_{L^{4}}^{4} d s} \varphi\left(X_{t}(x)\right)\right], \quad t \geq 0 .
$$

Now, consider the following equation

$$
d \tilde{X}_{t}=\mathscr{K}_{0}^{\prime} \tilde{X}_{t} d t+c|\cdot|_{L^{4}}^{4} \tilde{X}_{t} d t, \quad \tilde{X}_{0}=\varphi .
$$

Since $S_{t}$ is the corresponding semigroup of $\mathscr{K}_{0}^{\prime}$, there exists an unique mild solution

$$
\tilde{X}_{t}=S_{t} \varphi+c \int_{0}^{t} S_{t-s}\left(|\cdot|_{L^{4}}^{4} P_{s} \varphi\right) d s .
$$

Hence, $P_{t} \varphi$ is the solution of the Kolmogorov backward equation

$$
\frac{d P_{t} \varphi}{d t}=\mathscr{K}_{0} P_{t} \varphi=\mathscr{K}_{0}^{\prime} P_{t} \varphi+c|\cdot|_{L^{4}}^{4} P_{t} \varphi
$$

This yields

$$
P_{t} \varphi=S_{t} \varphi+c \int_{0}^{t} S_{t-s}\left(|\cdot|{ }_{L^{4}}^{4} P_{s} \varphi\right) d s .
$$

In order to get the estimate for $D P_{t} \varphi$, it is sufficient to estimate $D S_{t} \varphi$ by the above formula. To do this, we mainly follow the method developed in [4] for the Wiener case. We intend to extend it to the jump case. Because the proof is more involved, we need to check several crucial Propositions in [4] in jump case. The detailed steps are described below. 
For $h \in \mathbb{H}$, consider the following equation

$$
\left\{\begin{aligned}
d \eta_{t}^{h}(x)= & \left(A \eta_{t}^{h}(x)+\bar{b}\left(X_{t}(x)\right) \eta_{t}^{h}(x)\right) d t \\
& +\int_{U} D f(X(t-, x), u) \cdot \eta^{h}(t-, x) \tilde{N}(d t, d u), \\
\eta^{h}(0, x)= & h,
\end{aligned}\right.
$$

where $\bar{b}\left(X_{t}(x)\right) \eta_{t}^{h}(x)=D_{\xi}\left(X_{t}(x) \eta_{t}^{h}(x)\right)$. We can prove that there exists a unique solution $\eta_{t}^{h}(x)$ for this equation as in [9].

Lemma 4.1. Under the hypotheses $\left(\mathrm{H}_{1}\right)-\left(\mathrm{H}_{4}\right)$, for any $\alpha=-3 / 4,-1$, there exists $c=c(\alpha)>0$ such that

$$
\begin{aligned}
& \mathbb{E} e^{-c \int_{0}^{t}\left|X_{s}(x)\right|_{L^{4}}^{8 / 3} d s}\left|\eta_{t}^{h}(x)\right|_{\alpha}^{2}+\frac{1}{2} \mathbb{E} \int_{0}^{t} e^{-c \int_{0}^{s}|X(\tau, x)|_{L^{4}}^{8 / 3} d \tau}\left|\eta_{s}^{h}(x)\right|_{1+\alpha}^{2} d s \\
& \quad \leq e^{C_{f} t}|h|_{\alpha}^{2}
\end{aligned}
$$

Proof. We only prove the case of $\alpha=-3 / 4$, for $\alpha=-1$ is similar. Itô's formula yields

$$
\begin{aligned}
\left|(-A)^{\frac{\alpha}{2}} \eta_{t}^{h}(x)\right|_{\mathbb{H}}^{2}+2 \int_{0}^{t}\left|\eta_{s}^{h}(x)\right|_{1+\alpha}^{2} d s \\
\leq \mid \\
\quad\left|(-A)^{\frac{\alpha}{2}} h\right|_{\mathbb{H}}^{2}+2 \int_{0}^{t}\left|X_{s}(x)\right|_{L^{4}}\left|\eta_{s}^{h}(x)\right|_{L^{4}}\left|\eta_{s}^{h}(x)\right|_{1+2 \alpha} d s \\
\quad+2 \int_{0}^{t} \int_{U}\left\langle(-A)^{\alpha} \eta_{s-}^{h}(x), D f(X(t-, x), u) \eta_{s-}^{h}(x)\right\rangle \widetilde{N}(d s, d u) \\
\quad+\int_{0}^{t} \int_{U}\left\langle D f\left(X_{s-}(x), u\right) \eta_{s-}^{h}(x)\right. \\
\left.\quad(-A)^{\alpha} D f\left(X_{s-}(x), u\right), \eta_{s-}^{h}(x)\right\rangle N(d s, d u)
\end{aligned}
$$

By $\mathbb{H}^{\frac{1}{4}}(0,1) \subset L^{4}(0,1)$ and interpolation, one has

$$
\left|\eta_{t}^{h}(x)\right|_{L^{4}} \leq C\left|\eta_{t}^{h}(x)\right|_{\frac{1}{4}} \leq C\left|\eta_{t}^{h}(x)\right|_{\alpha}^{3 / 4+\alpha}\left|\eta_{t}^{h}(x)\right|_{1+\alpha}^{1 / 4-\alpha}
$$

and

$$
\left|\eta_{t}^{h}(x)\right|_{1+2 \alpha} \leq C\left|\eta_{t}^{h}(x)\right|_{\alpha}^{-\alpha}\left|\eta_{t}^{h}(x)\right|_{1+\alpha}^{1+\alpha} .
$$

These yield that there exists a constant $C_{\alpha}>0$ such that

$$
\begin{aligned}
& \left|X_{t}(x)\right|_{L^{4}}\left|\eta_{t}^{h}(x)\right|_{L^{4}}\left|\eta_{t}^{h}(x)\right|_{1+2 \alpha} \\
& \quad \leq C\left|X_{t}(x)\right|_{L^{4}}\left|\eta_{t}^{h}(x)\right|_{\alpha}^{3 / 4}\left|\eta_{t}^{h}(x)\right|_{1+\alpha}^{5 / 4} \\
& \quad \leq C_{\alpha}\left|X_{t}(x)\right|_{L^{4}}^{8 / 3}\left|\eta_{t}^{h}(x)\right|_{\alpha}^{2}+1 / 2\left|\eta_{t}^{h}(x)\right|_{1+\alpha}^{2}
\end{aligned}
$$


Using Itô's formula for $e^{-c(\alpha) \int_{0}^{t}\left|X_{s}(x)\right|_{L^{4}}^{8 / 3} d s}\left|\eta_{t}^{h}(x)\right|_{\alpha}^{2}$ with $c(\alpha) \triangleq 2 C_{\alpha}$, (4.11) and (4.12) imply

$$
\begin{aligned}
& \mathbb{E} e^{-c(\alpha) \int_{0}^{t}\left|X_{s}(x)\right|_{L^{4}}^{8 / 3} d s}\left|\eta_{t}^{h}(x)\right|_{\alpha}^{2}+\mathbb{E} \int_{0}^{t} e^{-c(\alpha) \int_{0}^{s}|X(\tau, x)|_{L^{4}}^{8 / 3} d \tau}\left|\eta_{s}^{h}(x)\right|_{1+\alpha}^{2} d s \\
& \leq|h|_{\alpha}^{2}+\mathbb{E} \int_{0}^{t} \int_{U} e^{-c(\alpha) \int_{0}^{s}|X(\tau, x)|_{L^{4}}^{8 / 3} d \tau}\left|D f\left(X_{s-}(x), u\right) \eta_{s}^{h}(x)\right|_{\alpha}^{2} \lambda(d u) d s \\
& \leq|h|_{\alpha}^{2}+C_{f} \mathbb{E} \int_{0}^{t} e^{-c(\alpha) \int_{0}^{s}|X(\tau, x)|_{L^{4}}^{8 / 3} d \tau}\left|\eta_{s}^{h}(x)\right|_{\alpha}^{2} d s .
\end{aligned}
$$

Gronwall's inequality shows that the result with $\alpha=-3 / 4$ holds.

LEMMA 4.2. For any $\varphi \in C_{b}(\mathbb{H}), S_{t} \varphi$ is differentiable in any direction $h \in \mathbb{H}$ and

$$
\begin{aligned}
& D S_{t} \varphi(x) h \\
& =\mathbb{E}\left[\varphi\left(X_{t}(x)\right) e^{-c \int_{0}^{t}\left|X_{s}(x)\right|_{L^{4}}^{4} d s} \int_{0}^{t}\left\langle\eta_{s}^{h}(x), d W_{s}\right\rangle\right] \\
& \quad-4 c \mathbb{E}\left[\varphi\left(X_{t}(x)\right) e^{-c \int_{0}^{t}\left|X_{s}(x)\right|_{L^{4}}^{4} d s} \int_{0}^{t}\left(1-\frac{s}{t}\right)\left\langle X_{s}^{3}(x), \eta_{s}^{h}(x)\right\rangle d s\right] .
\end{aligned}
$$

Proof. For fixed $t>0, \varphi \in C_{b}(\mathbb{H})$, define $u(t, x)=\mathbb{E} e^{-c \int_{0}^{t}\left|X_{s}(x)\right|_{L^{4}}^{4} d s} \times$ $\varphi\left(X_{t}(x)\right), 0 \leq s \leq t$. Itô's formula yields

$$
\begin{aligned}
e^{-c \int_{0}^{t}\left|X_{r}(x)\right|_{L^{4}}^{4} d r} \varphi\left(X_{t}(x)\right) \\
=u(t, x)+\int_{0}^{t}\left[D_{s} u\left(t-s, X_{s}(x)\right)+\mathscr{K}_{0}^{\prime} u\left(t-s, X_{s}(x)\right)\right] e^{-c \int_{0}^{s}\left|X_{r}(x)\right|_{L^{4}}^{4} d r} d s \\
\quad+\int_{0}^{t} \int_{U}\left[e ^ { - c \int _ { 0 } ^ { s } | X _ { r } ( x ) | _ { L ^ { 4 } } ^ { 4 } d r } \left(u\left(t-s, X_{s}(x)+f\left(X_{s-}(x), u\right)\right)\right.\right. \\
\left.\left.\quad-u\left(t-s, X_{s-}(x)\right)\right)\right] \tilde{N}(d s, d u) \\
\quad+\int_{0}^{t}\left\langle D_{x} u\left(t-s, X_{s}(x)\right) e^{-c \int_{0}^{s}\left|X_{r}(x)\right|_{L^{4}}^{4} d r}, d W_{s}\right\rangle \\
=u(t, x)+\int_{0}^{t}\left\langle D_{x} u\left(t-s, X_{s}(x)\right) e^{-c \int_{0}^{s}\left|X_{r}(x)\right|_{L^{4}}^{4} d r}, d W_{s}\right\rangle \\
\quad+\int_{0}^{t} \int_{U}\left[e ^ { - c \int _ { 0 } ^ { s } | X _ { r } ( x ) | _ { L ^ { 4 } } ^ { 4 } d r } \left(u\left(t-s, X_{s}(x)+f\left(X_{s-}(x), u\right)\right)\right.\right. \\
\left.\left.\quad-u\left(t-s, X_{s-}(x)\right)\right)\right] \tilde{N}(d s, d u) .
\end{aligned}
$$


Multiplying this identity by $\int_{0}^{t}\left\langle\eta_{s}^{h}(x), d W_{s}\right\rangle$ and taking the expectation, we obtain

$$
\begin{aligned}
& \mathbb{E}\left[\varphi\left(X_{t}(x)\right) e^{-c \int_{0}^{t}\left|X_{s}(x)\right|_{L^{4}}^{4} d s} \int_{0}^{t}\left\langle\eta_{s}^{h}(x), d W_{s}\right\rangle\right] \\
& \quad=\mathbb{E} \int_{0}^{t}\left\langle D_{x} u\left(t-s, X_{s}(x)\right), \eta_{s}^{h}(x)\right\rangle e^{-c \int_{0}^{s}\left|X_{r}(x)\right|_{L^{4}}^{4} d r} d s .
\end{aligned}
$$

On the other hand, since

$$
\begin{aligned}
D_{x}^{h}\left[e^{-c \int_{0}^{s}\left|X_{r}(x)\right|_{L^{4}}^{4} d r} u\left(t-s, X_{s}(x)\right)\right] \\
=-4 c e^{-c \int_{0}^{s}\left|X_{r}(x)\right|_{L^{4}}^{4} d r} u\left(t-s, X_{s}(x)\right) \int_{0}^{s}\left\langle X_{r}^{3}(x), \eta_{r}^{h}(x)\right\rangle d r \\
\quad+\left\langle D_{x} u\left(t-s, X_{s}(x)\right), \eta_{s}^{h}(x)\right\rangle e^{-c \int_{0}^{s}\left|X_{r}(x)\right|_{L^{4}}^{4} d r},
\end{aligned}
$$

we obtain

$$
\begin{aligned}
& \mathbb{E}\left[\varphi\left(X_{t}(x)\right) e^{-c \int_{0}^{t}\left|X_{s}(x)\right|_{L^{4}}^{4} d s} \int_{0}^{t}\left\langle\eta_{s}^{h}(x), d W_{s}\right\rangle\right] \\
&= \mathbb{E} \int_{0}^{t} D_{x}^{h}\left[e^{-c \int_{0}^{s}\left|X_{r}(x)\right|_{L^{4}}^{4} d r} u\left(t-s, X_{s}(x)\right)\right] d s \\
&+4 c \mathbb{E} \int_{0}^{t} e^{-c \int_{0}^{s}\left|X_{r}(x)\right|_{L^{4}}^{4} d r} \int_{0}^{s}\left\langle X_{r}^{3}(x), \eta_{r}^{h}(x)\right\rangle d r u\left(t-s, X_{s}(x)\right) d s \\
&= \int_{0}^{t} D^{h} S_{t} \varphi(x) d s \\
&+\mathbb{E}\left[\varphi\left(X_{t}(x)\right) e^{-c \int_{0}^{t}\left|X_{r}(x)\right|_{L^{4}}^{4} d r} \int_{0}^{t} \int_{0}^{s}\left\langle X_{r}^{3}(x), \eta_{r}^{h}(x)\right\rangle d r d s\right] \\
&= t D^{h} S_{t} \varphi(x) \\
&+4 c \mathbb{E}\left[\varphi\left(X_{t}(x)\right) e^{-c \int_{0}^{t}\left|X_{r}(x)\right|_{L^{4}}^{4} d r} \int_{0}^{t}\left(1-\frac{s}{t}\right)\left\langle X_{s}^{3}(x), \eta_{s}^{h}(x)\right\rangle d s\right],
\end{aligned}
$$

which implies the result.

LEMMA 4.3. Let $c$ be sufficiently large. For any $\varphi$ with $\|\varphi\|_{0, L^{4}, k}:=$ $\sup _{x \in L^{4}} \frac{|\varphi(x)|}{1+|x|_{L^{4}}^{k}}<\infty$, there is a constant $C>0$ such that

$$
\left|D S_{t} \varphi(x)\right|_{3 / 4} \leq C e^{C t}\left(1+t^{-7 / 8}\right)\left(|x|_{L^{4}}+1\right)^{k}\|\varphi\|_{0, L^{4}, k}, \quad x \in L^{4}, t>0 .
$$

Proof. Following the proof of Lemma 3.2 in [4], we can easily obtain the desired result by Lemmas 4.1 and 4.2. Since the proof is almost the same as that of Lemma 3.2 in [4], we omit it here. 
LEMMA 4.4. For $c$ being sufficiently large and for $\varphi \in C_{b}^{1}(\mathbb{H})$ with $\sup _{x \in \mathbb{H}}|D \varphi(x)|_{1} \leq C_{\varphi}$, there exists a constant $C>0$ such that

$$
\left|D S_{t} \varphi(x)\right|_{1} \leq C_{\varphi}+C\|\varphi\|_{0}\left(|x|_{L^{6}}+1\right)^{3} e^{C t} .
$$

Proof. By Hölder's inequality, Theorem 1.1 and Lemma 4.1 with $\alpha=-1$, we have

$$
\begin{aligned}
D S_{t} \varphi(x) h= & \mathbb{E}\left[e^{-c \int_{0}^{t}\left|X_{s}(x)\right|_{L^{4}}^{4} d s} D \varphi\left(X_{t}(x)\right) \cdot \eta_{s}^{h}(x)\right] \\
& -4 c \mathbb{E}\left[e^{-c \int_{0}^{t}\left|X_{r}(x)\right|_{L^{4}}^{4} d r} \int_{0}^{t}\left\langle X_{r}^{3}(x), \eta_{r}^{h}(x)\right\rangle d r \varphi\left(X_{t}(x)\right)\right] \\
\leq & C_{\varphi} \mathbb{E}\left[e^{-c \int_{0}^{t}\left|X_{s}(x)\right|_{L^{4}}^{4} d s}\left|\eta_{s}^{h}(x)\right|_{-1}\right] \\
& +C\|\varphi\|_{0} \mathbb{E}\left[\sup _{t \in[0, T]}\left|X_{t}(x)\right|_{L^{6}}^{3} \int_{0}^{t} e^{-c \int_{0}^{s}\left|X_{r}(x)\right|_{L^{4}}^{4} d r}\left|\eta_{s}^{h}(x)\right| d s\right] \\
\leq & \left(C_{\varphi}+C\|\varphi\|_{0}\left(|x|_{L^{6}}+1\right)^{3}\right) e^{C t}|h|_{-1} .
\end{aligned}
$$

This implies the result.

LEMmA 4.5. Assume $\varphi \in C_{b}^{1}(\mathbb{H})$ and $\sup _{x \in \mathbb{H}}|D \varphi(x)|_{1} \leq C_{\varphi}$. Then there exists a constant $C>0$ such that

$$
\left|D P_{t} \varphi(x)\right|_{3 / 4} \leq C_{\varphi}+C\|\varphi\|_{0} e^{C t}\left(|x|_{L^{6}}+1\right)^{4} .
$$

Proof. Let $h \in \mathbb{H}$, we have

$$
D P_{t} \varphi(x) h=D S_{t} \varphi(x) h+c \int_{0}^{t} D S_{t-s}\left(|\cdot|_{L^{4}}^{4} P_{s} \varphi\right)(x) h d s,
$$

where we choose $c$ such that Lemmas 4.3 and 4.4 hold. The inequality

$$
\left\||\cdot|{ }_{L^{4}}^{4} P_{s} \varphi\right\|_{0, L^{4}, 4}=\sup _{x \in \mathbb{H}} \frac{|x|_{L^{4}}^{4}\left|P_{s} \varphi(x)\right|}{1+|x|_{L^{4}}^{4}} \leq\|\varphi\|_{0},
$$

implies

$$
\begin{aligned}
\left|D P_{t} \varphi(x) h\right| \leq & \left(C_{\varphi}+C\|\varphi\|_{0}\left(|x|_{L^{6}}+1\right)^{3} e^{C t}\right)|h|_{-1} \\
& +C\|\varphi\|_{0} \int_{0}^{t} e^{C(t-s)}\left(1+(t-s)^{-7 / 8}\right)\left(|x|_{L^{4}}+1\right)^{4} d s|h|_{-3 / 4},
\end{aligned}
$$

which gives the result.

Now, we are going to extend Proposition 3.6 in [4] to the jump case.

Proposition 4.1. Under the conditions $\left(\mathrm{H}_{1}\right)-\left(\mathrm{H}_{4}\right)$, there exists $w_{1}>0$ such that, for $m \in \mathbb{N}, t>0$ and $\varphi \in C_{b}^{1}(\mathbb{H})$ with $D \varphi \in C_{b}\left(\mathbb{H}, \mathbb{H}^{1}(0,1)\right)$, we have $D P_{t}^{m} \varphi(x) \in \mathbb{H}^{1}(0,1)$ and

$$
\left|D P_{t}^{m} \varphi(x)\right|_{\mathbb{H}^{1}(0,1)} \leq\left(\|D \varphi\|_{C_{b}\left(\mathbb{H}, \mathbb{H}^{1}(0,1)\right)}+w_{1}\|\varphi\|_{0}\right)\left(1+|x|_{L^{6}}\right)^{8} e^{w_{1} t} .
$$


Proof. Let $h \in \mathbb{H}$, we have

$$
\begin{aligned}
& D P_{t} \varphi(x) h=D S_{t} \varphi(x) h+c \int_{0}^{t} D S_{t-s}\left(|\cdot|{ }_{L^{4}}^{4} P_{s} \varphi\right)(x) h d s \\
& =D S_{t} \varphi(x) h+4 c \int_{0}^{t} \mathbb{E}\left[e^{-c \int_{0}^{t-s}\left|X_{r}(x)\right|_{L^{4}}^{4} d r}\left\langle X^{3}(t-s, x), \eta_{t-s}^{h}(x)\right\rangle\right. \\
& \left.\times P_{s} \varphi\left(X_{t-s}(x)\right)\right] d s \\
& +c \int_{0}^{t} \mathbb{E}\left[e^{-c \int_{0}^{t-s}\left|X_{r}(x)\right|_{L^{4}}^{4} d r}\left|X_{t-s}(x)\right|_{L^{4}}^{4}\right. \\
& \left.\times D P_{s} \varphi\left(X_{t-s}(x)\right) \cdot \eta_{t-s}^{h}(x)\right] d s \\
& -4 c^{2} \int_{0}^{t} \mathbb{E}\left[e^{-c \int_{0}^{t-s}\left|X_{r}(x)\right|_{L^{4}}^{4} d r}\left|X_{t-s}(x)\right|_{L^{4}}^{4} P_{s} \varphi\left(X_{t-s}(x)\right)\right. \\
& \left.\times \int_{0}^{t-s}\left\langle X^{3}(r, x), \eta_{r}^{h}(x)\right\rangle d r\right] d s \\
& \hat{=} J_{1}+J_{2}+J_{3}+J_{4} \text {. }
\end{aligned}
$$

By Lemma 4.4, we get

$$
\left|D S_{t} \varphi(x)\right|_{1} \leq\|D \varphi\|_{C_{b}\left(\mathbb{H}, \mathbb{H}^{1}(0,1)\right)}+C\|\varphi\|_{0}\left(|x|_{L^{6}}+1\right)^{3} e^{C t}
$$

Theorem 1.1 and Lemma 4.1 with $\alpha=-1$ give

$$
\begin{aligned}
\left|J_{2}\right| & \leq 4 c\|\varphi\|_{0} \mathbb{E}\left(\sup _{t \in[0, T]}\left|X_{t}(x)\right|_{L^{6}}^{3} \int_{0}^{t} e^{-c \int_{0}^{t-s}\left|X_{r}(x)\right|_{L^{4}}^{4} d r}\left|\eta_{t-s}^{h}(x)\right| d s\right) \\
& \leq C\|\varphi\|_{0}\left(|x|_{L^{6}}+1\right)^{3}\left(\mathbb{E} \int_{0}^{t} e^{-2 c \int_{0}^{t-s}\left|X_{r}(x)\right|_{L^{4}}^{4} d r}\left|\eta_{t-s}^{h}(x)\right|^{2} d s\right)^{1 / 2} \\
& \leq C\|\varphi\|_{0}\left(|x|_{L^{6}}+1\right)^{3} e^{C t}|h|_{-1} .
\end{aligned}
$$

Set $D\left(X_{t-s}(x), \varphi\right)=\|D \varphi\|_{C_{b}\left(\mathbb{H}, \mathbb{H}^{1}(0,1)\right)}+C\|\varphi\|_{0} e^{s C}\left(\left|X_{t-s}(x)\right|_{L^{6}}+1\right)^{4}$. By Lemma 4.5 , Lemma 4.1 and $\left.|\cdot|\right|_{-3 / 4} \leq C|\cdot|_{0}$, one has

$$
\begin{aligned}
\left|J_{3}\right| \leq & c \mathbb{E}\left(\int_{0}^{t} e^{-c \int_{0}^{t-s}\left|X_{r}(x)\right|_{L^{4}}^{4} d r}\left|X_{t-s}(x)\right|_{L^{4}}^{4}\right. \\
& \left.\times D\left(X_{t-s}(x), \varphi\right)\left|\eta_{t-s}^{h}(x)\right|_{-3 / 4} d s\right) \\
\leq & C \mathbb{E}\left(\int_{0}^{t} e^{-c \int_{0}^{t-s}\left|X_{r}(x)\right|_{L^{4}}^{4} d r}\left|X_{t-s}(x)\right|_{L^{4}}^{4}\right. \\
& \left.\times D\left(X_{t-s}(x), \varphi\right)\left|\eta_{t-s}^{h}(x)\right|_{0} d s\right) \\
\leq & \left(\|D \varphi\|_{C_{b}\left(\mathbb{H}, \mathbb{H}^{1}(0,1)\right)}+C\|\varphi\|_{0}\right)\left(|x|_{L^{6}}+1\right)^{8} e^{C t}|h|_{-1} .
\end{aligned}
$$


$J_{4}$ can be treated similarly as $J_{2}$ and we can easily obtain

$$
\left|J_{4}\right| \leq C\|\varphi\|_{0}\left(|x|_{L^{6}}+1\right)^{8} e^{C t}|h|_{-1} .
$$

Hence, we deduce that there exits a constant $w_{1}>0$ such that

$$
\left|D P_{t} \varphi(x) h\right| \leq\left(\|D \varphi\|_{C_{b}\left(\mathbb{H}, \mathbb{H}^{1}(0,1)\right)}+w_{1}\|\varphi\|_{0}\right)\left(|x|_{L^{6}}+1\right)^{8} e^{w_{1} t}|h|_{-1} .
$$

The conclusion holds for $D P_{t} \varphi(x)$. We can then easily obtain that the conclusion also holds uniformly on $D P_{t}^{m} \varphi(x)$.

The following two results are essential for the proof of our main results.

Proposition 4.2. Take $\lambda>w_{0} \vee w_{1}$, where $w_{0}$ and $w_{1}$ are the same as in Theorem 3.2 and Proposition 4.1 respectively. For $g \in \mathscr{E}_{A}(\mathbb{H})$ and $m \geq 1$, define

$$
\varphi(x)=\int_{0}^{\infty} e^{-\lambda t} P_{t}^{m} g(x) d t, \quad x \in L^{6}(0,1) .
$$

Then (i) $\varphi$ is continuous, bounded and Fréchet differentiable and $D \varphi \in$ $C\left(L^{6}(0,1), \mathbb{H}^{1}(0,1)\right)$. Moreover, it holds

$$
|D \varphi(x)|_{\mathbb{H}^{1}(0,1)} \leq \frac{1}{\lambda-w_{1}}\left(\|D g\|_{C_{b}\left(\mathbb{H}, \mathbb{H}^{1}(0,1)\right)}+w_{1}\|g\|_{0}\right)\left(1+|x|_{L^{6}}\right)^{8} .
$$

(ii) For $\varphi \in \mathscr{D}\left(\mathscr{L}_{V}, C_{b, V}\left(L^{6}\right)\right) \cap \mathscr{D}\left(\mathscr{K}_{m}, C_{b, V}\left(L^{6}\right)\right)$ and $x \in L^{6}(0,1)$,

$$
\begin{aligned}
\mathscr{K}_{m} \varphi(x)= & \mathscr{L}_{V} \varphi(x)-\frac{1}{2}\left\langle D_{\xi} T_{m} D \varphi(x),\left(T_{m} x\right)^{\otimes 2}\right\rangle \\
& -\frac{1}{2} \operatorname{Tr}\left[D^{2} \varphi(x)\left(I-T_{m}\right) Q\right] \\
& +\int_{U}\left[\varphi\left(x+T_{m} f\left(T_{m} x, u\right)\right)-\varphi(x)\right. \\
& \left.-\left\langle D \varphi(x), T_{m} f\left(T_{m} x, u\right)\right\rangle\right] \lambda(d u) .
\end{aligned}
$$

Proof. (i) The proof is the same as that of Proposition 11 of [18].

(ii) Theorems 3.2 and 3.3 imply $\varphi=R\left(\lambda, \mathscr{K}_{m}\right) g$ and $\varphi \in \mathscr{D}\left(\mathscr{K}_{m}, C_{b, V}\left(L^{6}\right)\right)$. Then we only need to show $\varphi \in \mathscr{D}\left(\mathscr{L}_{V}, C_{b, V}\left(L^{6}\right)\right)$.

(a) Let $X_{t}^{m}$ be the solution of (4.2). By using Itô's formula and taking expectation, we have

$$
\begin{aligned}
\frac{\mathbb{E} \varphi\left(X_{t}^{m}(x)\right)-\varphi(x)}{t}= & \frac{1}{t} \mathbb{E} \int_{0}^{t}\left\langle D \varphi\left(X_{s}^{m}\right), A X_{s}^{m}+b_{m} X_{s}^{m}\right\rangle d s \\
& +\frac{1}{2 t} \mathbb{E} \int_{0}^{t} \operatorname{Tr}\left(D^{2} \varphi\left(X_{s}^{m}\right) T_{m} Q\right) d s \\
& +\frac{1}{t} \mathbb{E} \int_{0}^{t} \int_{U}\left[\varphi\left(X_{s}^{m}+T_{m} f\left(X_{s}^{m}, u\right)\right)-\varphi\left(X_{s}^{m}\right)\right. \\
& \left.-\left\langle D \varphi\left(X_{s}^{m}\right), T_{m} f\left(X_{s}^{m}, u\right)\right\rangle\right] \lambda(d u) d s
\end{aligned}
$$


We shall prove the existence of the limit of each term in the right-hand side of (4.14).

By (1.1) and the definition of mild solution, one has that, for $0 \leq s \leq T$ and $x \in \mathscr{D}(A) \cap L^{6}(0,1)$,

$$
\begin{aligned}
& \left|A X_{s}^{m}(x)\right|_{\mathbb{H}} \\
& \leq\left|A e^{s A} x\right|_{\mathbb{H}}+\mid A\left[\frac{1}{2} \int_{0}^{s} e^{(s-\tau) A} T_{m} D_{\xi}\left(T_{m} X_{\tau}^{m}(x)\right)^{2} d \tau+W_{A}^{m}(s)\right. \\
& \left.\quad+\int_{0}^{s} \int_{U} e^{(s-\tau) A} T_{m} f\left(T_{m} X_{\tau-}^{m}(x), u\right) \tilde{N}(d \tau, d u)\right]\left.\right|_{\mathbb{H}_{m}} \\
& \leq e^{s \gamma_{2}}|A x|_{\mathbb{H}}+\left|\lambda_{m}\right| \mid \int_{0}^{s} e^{(s-\tau) A} T_{m} D_{\xi}\left(T_{m} X_{\tau}^{m}(x)\right)^{2} d \tau+W_{A}^{m}(s) \\
& \quad+\left.\int_{0}^{s} \int_{U} e^{(s-\tau) A} T_{m} f\left(T_{m} X_{\tau-}^{m}(x), u\right) \tilde{N}(d \tau, d u)\right|_{\mathbb{H}_{m}} \\
& \leq e^{s \gamma_{2}}|A x|_{\mathbb{H}}+\left|\lambda_{m}\right| \cdot\left|X_{s}^{m}(x)-e^{s A} x\right|_{\mathbb{H}} \\
& \leq e^{\gamma_{2} T}|A x|_{\mathbb{H}}+\left|\lambda_{m}\right|\left(\left|X_{s}^{m}(x)\right|_{L^{2}}+e^{\gamma_{2} T}|x|_{\mathbb{H}}\right)
\end{aligned}
$$

and

$$
\left|b_{m} X_{s}^{m}\right|_{\mathbb{H}_{m}} \leq C\left|T_{m} X_{s}^{m}\right|_{\mathbb{H}^{1}}^{2} \leq C\left|\lambda_{m}\right|\left|T_{m} X_{s}^{m}\right|_{\mathbb{H}_{m}}^{2} \leq C\left|\lambda_{m}\right|\left|X_{s}^{m}\right|_{\mathbb{H}^{*}}^{2} .
$$

Similarly, one also has

$$
\begin{aligned}
& \left|A\left(X_{s}^{m}(x)-X_{s^{\prime}}^{m}(x)\right)\right|_{\mathbb{H}} \\
& \quad \leq\left|\left(e^{s A}-e^{s^{\prime} A}\right) A x\right|_{\mathbb{H}}+\left|\lambda_{m}\right|\left|X_{s}^{m}(x)-X_{s^{\prime}}^{m}(x)\right|_{\mathbb{H}} \\
& \quad+\left|\lambda_{m}\right|\left|\left(e^{s A}-e^{s^{\prime} A}\right) x\right|_{\mathbb{H}}
\end{aligned}
$$

and

$$
\begin{aligned}
& \left|b_{m} X_{s}^{m}-b_{m} X_{s^{\prime}}^{m}\right|_{\mathbb{H}} \\
& \quad \leq C\left|T_{m} X_{s}^{m}-T_{m} X_{s^{\prime}}^{m}\right|_{\mathbb{H}^{1}}\left(\left|T_{m} X_{s}^{m}\right|_{\mathbb{H}^{1}}+\left|T_{m} X_{s^{\prime}}^{m}\right|_{\mathbb{H}^{1}}\right) \\
& \quad \leq C\left|\lambda_{m}\right|\left|X_{s}^{m}-X_{s^{\prime}}^{m}\right|_{\mathbb{H}}\left(\left|X_{s}^{m}\right|_{\mathbb{H}}+\left|X_{s^{\prime}}^{m}\right|_{\mathbb{H}}\right) .
\end{aligned}
$$

Itô's formula implies that, for $s>s^{\prime} \geq 0$,

$$
\begin{aligned}
\mid X_{s}^{m} & -\left.X_{s^{\prime}}^{m}\right|_{\mathbb{H}} ^{2} \\
= & 2 \int_{s^{\prime}}^{s}\left\langle X_{\tau}^{m}-X_{s^{\prime}}^{m}, b_{m}\left(X_{\tau}^{m}\right)\right\rangle d \tau \\
& +2 \int_{s^{\prime}}^{s} \int_{U}\left\langle X_{\tau-}^{m}-X_{s^{\prime}}^{m}, T_{m} f\left(X_{\tau-}^{m}, u\right)\right\rangle \tilde{N}(d \tau, d u) \\
& +\int_{s^{\prime}}^{s} \int_{U}\left|T_{m} f\left(T_{m} X_{\tau-}^{m}, u\right)\right|_{\mathbb{H}}^{2} N(d \tau, d u) .
\end{aligned}
$$


It is well known that

$$
\mathbb{E}\left|W_{A}^{m}(s)-W_{A}^{m}\left(s^{\prime}\right)\right|_{\mathbb{H}}^{2} \rightarrow 0, \quad s \rightarrow s^{\prime} .
$$

By (4.19) and (4.20), one has

$$
\mathbb{E}\left|X_{s}^{m}-X_{s^{\prime}}^{m}\right|_{\mathbb{H}}^{2} \rightarrow 0, \quad s \rightarrow s^{\prime}
$$

Similarly, we can prove

$$
\mathbb{E}\left|X_{s}^{m}-X_{s^{\prime}}^{m}\right|_{\mathbb{H}}^{4} \rightarrow 0, \quad s \rightarrow s^{\prime} .
$$

By Proposition 4.2(i), for $g(x)=e^{i\langle x, h\rangle}$ with $h \in \mathbb{H}^{1}(0,1)$, we have

$$
\begin{aligned}
\mid D \varphi & \left(X_{s}^{m}\right)-\left.D \varphi\left(X_{s^{\prime}}^{m}\right)\right|_{\mathbb{H}^{1}(0,1)} \\
\leq & \frac{1}{\lambda-w_{1}}\left[\| D g\left(\cdot+X_{s}^{m}-X_{s^{\prime}}^{m}\right)-\left.D g(\cdot)\right|_{C_{b}\left(\mathbb{H}, \mathbb{H}^{1}(0,1)\right)}\right. \\
& \left.+C \| g\left(\cdot+X_{s}^{m}-X_{s^{\prime}}^{m}\right)-\left.g(\cdot)\right|_{0}\right]\left[1+\left|X_{s^{\prime}}^{m}\right|_{L^{6}}\right]^{8} \\
= & \frac{1}{\lambda-w_{1}}\left[\sup _{x \in \mathbb{H}}\left|e^{i\left\langle x+X_{s}^{m}-X_{s^{\prime}}^{m}, h\right\rangle} h-e^{i\langle x, h\rangle} h\right|_{\mathbb{H}^{1}(0,1)}\right. \\
& \left.+C \sup _{x \in \mathbb{H}^{1}}\left|e^{i\left\langle x+X_{s}^{m}-X_{s^{\prime}}^{m}, h\right\rangle}-e^{i\langle x, h\rangle}\right|\right]\left[1+\left|X_{s^{\prime}}^{m}\right|_{L^{6}}\right]^{8} \\
\leq & \frac{1}{\lambda-w_{1}}\left(|h|_{\mathbb{H}^{1}(0,1)}^{2}\left|X_{s}^{m}-X_{s^{\prime}}^{m}\right|_{\mathbb{H}^{-1}(0,1)}\right. \\
& \left.+C|h|_{\mathbb{H}^{1}(0,1)}\left|X_{s}^{m}-X_{s^{\prime}}^{m}\right|_{\mathbb{H}^{-1}(0,1)}\right)\left(1+\left|X_{s^{\prime}}^{m}\right|_{L^{6}}\right)^{8} \\
\leq & C\left(|h|_{\mathbb{H}^{1}(0,1)}^{2}\left|X_{s}^{m}-X_{s^{\prime}}^{m}\right|_{\mathbb{H}^{1}}+|h|_{\mathbb{H}^{1}(0,1)}\left|X_{s}^{m}-X_{s^{\prime}}^{m}\right|_{\mathbb{H}}\right)\left(1+\left|X_{s^{\prime}}^{m}\right|_{L^{6}}\right)^{8} .
\end{aligned}
$$

Theorem 1.1 and (4.22) show

$$
\begin{aligned}
& \mathbb{E} \mid D \varphi\left(X_{s}^{m}\right)-\left.D \varphi\left(X_{s^{\prime}}^{m}\right)\right|_{\mathbb{H}} ^{2} \\
& \leq \mathbb{E}\left|D \varphi\left(X_{s}^{m}\right)-D \varphi\left(X_{s^{\prime}}^{m}\right)\right|_{\mathbb{H}^{1}(0,1)}^{2} \\
& \quad \leq C \mathbb{E}\left[\left|X_{s}^{m}-X_{s^{\prime}}^{m}\right|_{\mathbb{H}^{-1}(0,1)}^{2}\left(1+\left|X_{s^{\prime}}^{m}\right|_{L^{6}}\right)^{16}\right] \\
& \quad \leq C\left[\mathbb{E}\left|X_{s}^{m}-X_{s^{\prime}}^{m}\right|_{\mathbb{H}}^{4}\right]^{1 / 2}\left[\mathbb{E}\left(1+\left|X_{s^{\prime}}^{m}\right|_{L^{6}}\right)^{32}\right]^{1 / 2} \rightarrow 0, \quad s \rightarrow s^{\prime}
\end{aligned}
$$

By Theorem 1.1 and (4.15)-(4.23), we have

$$
\begin{aligned}
\mid \mathbb{E}\langle & \left.D \varphi\left(X_{s}^{m}\right), A X_{s}^{m}+b_{m} X_{s}^{m}\right\rangle-\mathbb{E}\left\langle D \varphi\left(X_{s^{\prime}}^{m}\right), A X_{s^{\prime}}^{m}+b_{m} X_{s^{\prime}}^{m}\right\rangle \mid \\
\leq & \mathbb{E}\left(\left|D \varphi\left(X_{s}^{m}\right)-D \varphi\left(X_{s^{\prime}}^{m}\right)\right|_{\mathbb{H}}\left|A X_{s}^{m}+b_{m} X_{s}^{m}\right|_{\mathbb{H}}\right) \\
& +\mathbb{E}\left(\left|D \varphi\left(X_{s^{\prime}}^{m}\right)\right|_{\mathbb{H}^{1}}\left|A\left(X_{s}^{m}-X_{s^{\prime}}^{m}\right)+b_{m} X_{s}^{m}-b_{m} X_{s^{\prime}}^{m}\right|_{\mathbb{H}-1}\right) \\
\leq & C\left[\mathbb{E}\left|D \varphi\left(X_{s}^{m}\right)-D \varphi\left(X_{s^{\prime}}^{m}\right)\right|_{\mathbb{H}}^{2}\right]^{1 / 2}\left[\mathbb{E}\left(\left|A X_{s}^{m}\right|_{\mathbb{H}}^{2}+\left|b_{m} X_{s}^{m}\right|_{\mathbb{H}}^{2}\right)\right]^{1 / 2} \\
& +\left(\mathbb{E}\left|D \varphi\left(X_{s^{\prime}}^{m}\right)\right|_{\mathbb{H}^{1}}^{2}\right)^{1 / 2}\left[\mathbb { E } \left(2\left|A\left(X_{s}^{m}-X_{s^{\prime}}^{m}\right)\right|_{\mathbb{H}-1}^{2}\right.\right. \\
& \left.\left.+2\left|b_{m} X_{s}^{m}-b_{m} X_{s^{\prime}}^{m}\right|_{\mathbb{H}-1}^{2}\right)\right]^{1 / 2} \rightarrow 0, \quad s \rightarrow s^{\prime} .
\end{aligned}
$$


(4.24) yields

$$
\lim _{t \rightarrow 0^{+}} \frac{1}{t} \mathbb{E} \int_{0}^{t}\left\langle D \varphi\left(X_{s}^{m}\right), A X_{s}^{m}+b_{m} X_{s}^{m}\right\rangle d s=\left\langle D \varphi(x), A x+b_{m} x\right\rangle .
$$

For $k, l \in \mathbb{H}$, we have

$$
\begin{aligned}
\mathbb{E}\left[D^{2} g\left(X_{t}^{m}(x)\right)(k, l)\right]= & -\mathbb{E}\left(e^{i\left\langle X_{t}^{m}(x), h\right\rangle}\left\langle h, \eta_{m, t}^{k}(x)\right\rangle\left\langle h, \eta_{m, t}^{l}(x)\right\rangle\right) \\
& +i \mathbb{E}\left[e^{i\left\langle X_{t}^{m}(x), h\right\rangle}\left\langle h, \zeta_{m, t}^{k, l}(x)\right\rangle\right],
\end{aligned}
$$

where $\eta_{m, t}^{k}(x)=D X_{t}^{m}(x) k$ is the solution of the equation

$$
\left\{\begin{aligned}
d \eta_{m, t}^{k}(x)= & A \eta_{m, t}^{k}(x) d t+D_{\xi}\left(X_{t}^{m}(x) \eta_{m, t}^{k}(x)\right) d t \\
& +\int_{U} D T_{m} f\left(X_{t-}^{m}(x), u\right) \eta_{m, t-}^{k}(x) \widetilde{N}(d t, d u), \\
\eta_{m, 0}^{k}(x)= & \langle k, x\rangle
\end{aligned}\right.
$$

and $\zeta_{m, t}^{k, l}(x)=D^{2} X_{t}^{m}(x)(k, l)$ is the solution of the equation

$$
\left\{\begin{aligned}
d \zeta_{m, t}^{k, l}(x)= & A \zeta_{m, t}^{k, l}(x) d t+D_{\xi}\left(\eta_{m, t}^{k}(x) \cdot \eta_{m, t}^{l}(x)+X_{t}^{m}(x) \zeta_{m, t}^{k, l}(x)\right) d t \\
& +\int_{U}\left[D T_{m} f\left(X_{t-}^{m}(x), u\right) \zeta_{m, t-}^{k, l}(x)+D^{2} T_{m} f\left(X_{t-}^{m}(x), u\right)\right. \\
& \left.\times\left(\eta_{m, t-}^{k}(x), \eta_{m, t-}^{k}(x)\right)\right] \widetilde{N}(d t, d u), \\
\zeta_{m, 0}^{k, l}(x)= & 0 .
\end{aligned}\right.
$$

By Itô's formula and Gronwall's inequality, we can prove that $\mathbb{E}\left[i e^{i\left\langle X_{t}^{m}(\cdot), h\right\rangle}\langle h\right.$, $\left.\left.\zeta_{m, t}^{k, l}(\cdot)\right\rangle\right]$ and $\mathbb{E}\left(-e^{i\left\langle X_{t}^{m}(\cdot), h\right\rangle}\left\langle h, \eta_{m, t}^{k}(\cdot)\right\rangle\left\langle h, \eta_{m, t}^{l}(\cdot)\right\rangle\right)$ are continuous in $\mathbb{H}$. Hence, we have that

$\operatorname{Tr}\left[D^{2} \varphi(\cdot) T_{m} Q\right]=\sum_{k=1}^{m}\left\langle D^{2} \varphi(\cdot) Q e_{k}, e_{k}\right\rangle=\sum_{k=1}^{m} \int_{0}^{\infty} e^{-\lambda t}\left\langle D^{2} P_{t}^{m} g(\cdot) Q e_{k}, e_{k}\right\rangle d t$ implies

$$
\lim _{t \rightarrow 0^{+}} \frac{1}{2 t} \mathbb{E} \int_{0}^{t} \operatorname{Tr}\left(D^{2} \varphi\left(X_{s}^{m}\right) T_{m} Q\right) d s=\frac{1}{2} \operatorname{Tr}\left[D^{2} \varphi(x) T_{m} Q\right] .
$$

For $0 \leq s<s^{\prime}$, the conditions $\left(\mathrm{H}_{1}\right)$ and $\left(\mathrm{H}_{2}\right)$ show

$$
\begin{aligned}
\mid \mathbb{E} \int_{U} & {\left[\left\langle D \varphi\left(X_{s}^{m}\right), T_{m} f\left(X_{s}^{m}, u\right)\right\rangle-\left\langle D \varphi\left(X_{s^{\prime}}^{m}\right), T_{m} f\left(X_{s^{\prime}}^{m}, u\right)\right\rangle\right] \lambda(d u) \mid } \\
\leq & \mathbb{E} \int_{U}\left[\left|\left\langle D \varphi\left(X_{s}^{m}\right)-D \varphi\left(X_{s^{\prime}}^{m}\right), T_{m} f\left(X_{s}^{m}, u\right)\right\rangle\right|\right. \\
& \left.\quad-\left|\left\langle D \varphi\left(X_{s^{\prime}}^{m}\right), T_{m} f\left(X_{s}^{m}, u\right)-T_{m} f\left(X_{s^{\prime}}^{m}, u\right)\right\rangle\right|\right] \lambda(d u) \\
\leq & C \mathbb{E}\left|D \varphi\left(X_{s}^{m}\right)-D \varphi\left(X_{s^{\prime}}^{m}\right)\right|_{\mathbb{H}}\left(1+\left|X_{s}^{m}\right|_{\mathbb{H}}\right) \\
& +C \mathbb{E}\left|D \varphi\left(X_{s^{\prime}}^{m}\right)\right|_{\mathbb{H}}\left|X_{s}^{m}-X_{s^{\prime}}^{m}\right|_{\mathbb{H}} \\
\leq & C\left(\mathbb{E}\left|D \varphi\left(X_{s}^{m}\right)-D \varphi\left(X_{s^{\prime}}^{m}\right)\right|_{\mathbb{H}}^{2}\right)^{1 / 2}\left(\mathbb{E}\left(1+\left|X_{s}^{m}\right|_{\mathbb{H}}^{2}\right)\right)^{1 / 2} \\
& +C\left(\mathbb{E}\left|D \varphi\left(X_{s^{\prime}}^{m}\right)\right|_{\mathbb{H}}^{2}\right)^{1 / 2}\left(\mathbb{E}\left|X_{s}^{m}-X_{s^{\prime}}^{m}\right|_{\mathbb{H}}^{2}\right)^{1 / 2},
\end{aligned}
$$


which goes to 0 as $s \rightarrow s^{\prime}$ by (4.21) and (4.23). Notice that

$$
\begin{aligned}
\varphi & \left(X_{s}^{m}+T_{m} f\left(X_{s}^{m}, u\right)\right)-\varphi\left(X_{s}^{m}\right) \\
& =\left\langle\int_{0}^{1} D \varphi\left(X_{s}^{m}+\nu T_{m} f\left(X_{s}^{m}, u\right)\right) d \nu, T_{m} f\left(X_{s}^{m}, u\right)\right\rangle .
\end{aligned}
$$

As the above, we can easily obtain

$$
\begin{aligned}
& \mid \mathbb{E} \int_{U}\left[\varphi\left(X_{s}^{m}+T_{m} f\left(X_{s}^{m}, u\right)\right)-\varphi\left(X_{s}^{m}\right)\right. \\
& \left.\quad-\varphi\left(X_{s^{\prime}}^{m}+T_{m} f\left(X_{s^{\prime}}^{m}, u\right)\right)-\varphi\left(X_{s^{\prime}}^{m}\right)\right] \lambda(d u) \mid \rightarrow 0
\end{aligned}
$$

as $s \rightarrow s^{\prime}$. Therefore, we obtain

$$
\begin{aligned}
\lim _{t \rightarrow 0^{+}} & \frac{1}{t} \mathbb{E} \int_{0}^{t} \int_{U}\left[\varphi\left(X_{s}^{m}+T_{m} f\left(X_{s}^{m}, u\right)\right)-\varphi\left(X_{s}^{m}\right)\right. \\
& \left.-\left\langle D \varphi\left(X_{s}^{m}\right), T_{m} f\left(X_{s}^{m}, u\right)\right\rangle\right] \lambda(d u) d s \\
= & \int_{U}\left[\varphi\left(x+T_{m} f\left(T_{m} x, u\right)\right)-\varphi(x)-\left\langle D \varphi(x), T_{m} f\left(T_{m} x, u\right)\right\rangle\right] \lambda(d u) .
\end{aligned}
$$

(4.14) and (4.25)-(4.27) show for $x \in \mathscr{D}(A) \cap L^{6}(0,1)$,

$$
\begin{aligned}
\mathscr{K}_{m} \varphi(x)= & \left\langle D \varphi(x), A x+b_{m} x\right\rangle+\frac{1}{2} \operatorname{Tr}\left[T_{m} Q D^{2} \varphi(x)\right] \\
& +\int_{U} \varphi\left(x+T_{m} f\left(T_{m} x, u\right)\right)-\varphi(x)-\left\langle D \varphi(x), T_{m} f\left(T_{m} x, u\right)\right\rangle \lambda(d u) .
\end{aligned}
$$

(b) In the following, we will prove $\varphi \in \mathscr{D}\left(\mathscr{L}_{V}, C_{b, V}\left(L^{6}\right)\right)$ and

$$
\mathscr{L}_{V} \varphi(x)=\langle D \varphi(x), A x\rangle+\frac{1}{2} \operatorname{Tr}\left[Q D^{2} \varphi(x)\right], \quad x \in \mathscr{D}(A) \cap L^{6}(0,1) .
$$

This yields

$$
\begin{aligned}
\mathscr{K}_{m} \varphi(x) \\
=\mathscr{L}_{V} \varphi(x)+\left\langle D \varphi(x), b_{m}(x)\right\rangle-\frac{1}{2} \operatorname{Tr}\left[\left(I-T_{m}\right) Q D^{2} \varphi(x)\right] \\
\quad+\int_{U}\left[\varphi\left(x+T_{m} f\left(T_{m} x, u\right)\right)-\varphi(x)-\left\langle D \varphi(x), T_{m} f\left(T_{m} x, u\right)\right\rangle\right] \lambda(d u) .
\end{aligned}
$$

For the proof of $\varphi \in \mathscr{D}\left(\mathscr{L}_{V}, C_{b, V}\left(L^{6}(0,1)\right)\right)$, we have to show the existence of the derivative $\frac{d}{d t} R_{t} \varphi(x)$ at $t=0$ and $\sup _{t \in(0,1)}\left\|R_{t} \varphi-\varphi\right\|_{0, V} / t<+\infty$, where $R_{t} \varphi(x)=\mathbb{E}\left[\varphi\left(Z_{t}(x)\right)\right]$.

Because the operator $A$ is unbounded, we now consider the approximate problem

$$
\left\{\begin{array}{l}
d Z_{t}^{n}=A_{n} Z_{t}^{n} d t+\sqrt{Q} d W_{t}, \quad t>0, \\
Z_{0}^{n}=x
\end{array}\right.
$$


where $A_{n}, n \geq 1$ are the Yosida approximations of $A$. Clearly the problem above has a unique solution $Z_{t}^{n}(x)$.

Put $R_{t}^{n} \varphi(x)=\mathbb{E}\left[\varphi\left(Z_{t}^{n}(x)\right)\right]$. Itô's formula yields

$$
\left.\frac{d}{d t} R_{t}^{n} \varphi(x)\right|_{t=0}=\lim _{t \rightarrow 0^{+}} \frac{R_{t}^{n} \varphi(x)-\varphi(x)}{t}=\left\langle A_{n} x, D \varphi(x)\right\rangle+\frac{1}{2}\left[\operatorname{Tr} Q D^{2} \varphi(x)\right] .
$$

Proposition 7.5 of [6] implies that

$$
\lim _{n \rightarrow \infty} Z_{t}^{n}(x)=Z_{t}(x), \quad \mathbb{P} \text {-a.s. }
$$

uniformly in $t \in[0, T]$. This shows

$$
\left.\lim _{n \rightarrow \infty} \frac{d}{d t} R_{t}^{n} \varphi(x)\right|_{t=0}=\left.\frac{d}{d t} R_{t} \varphi(x)\right|_{t=0} .
$$

Thus, it follows that (4.28) holds. $\sup _{t \in(0,1)}\left\|R_{t} \varphi(x)-\varphi(x)\right\|_{0, V} / t<\infty$ can be verified by (1.1) and Theorem 1.1. Then $\varphi \in \mathscr{D}\left(\mathscr{L}_{V}, C_{b, V}\left(L^{6}\right)\right)$ and (4.13) is established.

Proposition 4.3. Fixed $m \in \mathbb{N}, f \in \mathscr{E}_{A}(\mathbb{H})$, let $\varphi$ be as in Proposition 4.2. There exist $k \in \mathbb{N}$ and a $k$-indexed sequence $\left\{\varphi_{n_{1}, \ldots, n_{k}}\right\}_{\left(n_{1}, \ldots, n_{k}\right) \in \mathbb{N}^{k}} \subset \mathscr{E}_{A}(\mathbb{H})$ such that

$$
\begin{gathered}
\lim _{n_{1} \rightarrow \infty} \cdots \lim _{n_{k} \rightarrow \infty} \frac{\varphi_{n_{1}, \ldots, n_{k}}}{1+V} \stackrel{\pi}{=} \frac{\varphi}{1+V}, \\
\lim _{n_{1} \rightarrow \infty} \cdots \lim _{n_{k} \rightarrow \infty} \frac{\mathscr{L}_{0} \varphi_{n_{1}, \ldots, n_{k}}}{1+V} \stackrel{\pi}{=} \frac{\mathscr{L} \varphi}{1+V}, \\
\lim _{n_{1} \rightarrow \infty} \cdots \lim _{n_{k} \rightarrow \infty} \frac{\left\langle D_{\xi} D \varphi_{n_{1}, \ldots, n_{k}}, h\right\rangle}{1+|\cdot|_{L^{6}}^{8}} \stackrel{\pi}{=} \frac{\left\langle D_{\xi} D \varphi, h\right\rangle}{1+|\cdot|_{L^{6}}^{8}}, \quad h \in \mathbb{H} .
\end{gathered}
$$

Proof. The idea of the proof comes from Proposition 12 of [18]. But for the function

$$
\psi_{p}(x) \hat{=}\left(1+p^{-1}\left|e^{p^{-1} A} x\right|_{L^{6}}^{8}\right)^{-1} \varphi\left(e^{p^{-1} A} x\right), \quad x \in \mathbb{H}, p \in \mathbb{N},
$$

we need the inequality

$$
\left|\left\langle D \psi_{p}(x), h\right\rangle\right| \leq C\left(C_{p}\left(\lambda-w_{1}\right)^{-1}+8\|\varphi\|_{0}\right)\left(1+p^{1 / 2}\right)|h|_{\mathbb{H}},
$$

for constants $C_{p}>0$ and $C>0$. This inequality is different from that in [18].

Note that $\psi_{p}(x)$ is Fréchet differentiable with respect to $x \in \mathbb{H}$ and its differential $D \psi_{p}: \mathbb{H} \rightarrow \mathbb{H}$ is bounded continuous. Therefore, $D \psi_{p} \in C_{b}^{1}(\mathbb{H})$. Indeed, from Sobolev's embedding theorem, the interpolatory inequality and (1.2), we have

$$
\left|e^{t A} h\right|_{L^{6}} \leq\left|e^{t A} h\right|_{\mathbb{H}^{\frac{1}{3}}} \leq\left|e^{t A} h\right|_{\mathbb{H}^{1}} \leq C\left(1+t^{-1 / 2}\right)|h|_{\mathbb{H}} \cdot
$$


Hence, we obtain that there exists a constant $C_{g}>0$ such that

$$
\begin{aligned}
\left|\left\langle D \psi_{p}(x), h\right\rangle\right| & \leq \frac{p\left|D \varphi\left(e^{p^{-1} A} x\right)\right|_{\mathbb{H}}\left|e^{p^{-1} A} h\right|_{\mathbb{H}}}{p+\left|e^{p^{-1} A} x\right|_{L^{6}}^{8}}+\frac{8 p\|\varphi\|_{0}\left|e^{p^{-1} A} x\right|_{L^{6}}^{7}\left|e^{p^{-1} A} h\right|_{L^{6}}}{\left(p+\left|e^{p^{-1} A} x\right|_{L^{6}}^{8}\right)^{2}} \\
& \leq\left(\frac{p C_{g}\left(1+\left|e^{p^{-1} A} x\right|_{L^{6}}^{8}\right)}{\left(p+\left|e^{p^{-1} A} x\right|_{L^{6}}^{8}\right)\left(\lambda-w_{1}\right)}+\frac{8 p\|\varphi\| \|_{0}\left|e^{p^{-1} A} x\right|_{L^{6}}^{7}}{\left(p+\left|e^{p^{-1} A} x\right|_{L^{6}}^{8}\right)^{2}}\right)\left|e^{p^{-1} A} h\right|_{L^{6}} \\
& \leq\left(p C_{g}\left(\lambda-w_{1}\right)^{-1}+8\|\varphi\|_{0}\right) C\left(1+p^{1 / 2}\right)|h|_{\mathbb{H} \mathbb{1}}
\end{aligned}
$$

This is (4.29). The rest of the proof is the same as did in [18]. We omit it here.

\section{Main results}

We are ready to prove our main results.

Theorem 5.1. Suppose that the conditions $\left(\mathrm{H}_{1}\right)-\left(\mathrm{H}_{4}\right)$ hold.

(i) The operator $\left(\mathscr{K}, \mathscr{D}\left(\mathscr{K}, C_{b, V}\left(L^{6}\right)\right)\right)$ is the extension of $\mathscr{K}_{0}$, and for any $\varphi \in \mathscr{E}_{A}(\mathbb{H})$ we have $\varphi \in \mathscr{D}\left(\mathscr{K}, C_{b, V}\left(L^{6}\right)\right)$ and $\mathscr{K} \varphi=\mathscr{K}_{0} \varphi$.

(ii) $\mathscr{E}_{A}(\mathbb{H})$ is a $\pi$-core for $\left(\mathscr{K}, \mathscr{D}\left(\mathscr{K}, C_{b, V}\left(L^{6}\right)\right)\right)$, that is, for any $\varphi \in \mathscr{D}\left(\mathscr{K}, C_{b, V}\left(L^{6}\right)\right)$ there exist $m$ and an $m$-indexed sequence $\left\{\varphi_{n_{1}, \ldots, n_{k}}\right\}_{\left(n_{1}, \ldots, n_{k}\right) \in \mathbb{N}^{k}} \subset \mathscr{E}_{A}(\mathbb{H})$ such that

$$
\begin{array}{r}
\lim _{n_{1} \rightarrow \infty} \cdots \lim _{n_{m} \rightarrow \infty} \frac{\varphi_{n_{1}, \ldots, n_{m}}}{1+V} \stackrel{\pi}{=} \frac{\varphi}{1+V}, \\
\lim _{n_{1} \rightarrow \infty} \cdots \lim _{n_{m} \rightarrow \infty} \frac{\mathscr{K}_{0} \varphi_{n_{1}, \ldots, n_{m}}}{1+V} \stackrel{\pi}{=} \frac{\mathscr{K} \varphi}{1+V} .
\end{array}
$$

Proof. (i) For $h \in \mathscr{D}(A)$, it is sufficient to show the claim for $\varphi(x)=$ $e^{i\langle x, h\rangle}, x \in L^{6}(0,1)$. By using Itô formula and taking expectation, we have

$$
\begin{aligned}
\frac{\mathbb{E} \varphi\left(X_{t}(x)\right)-\varphi(x)}{t} \\
=\frac{1}{t} \mathbb{E} \int_{0}^{t}\left\langle A D \varphi\left(X_{s}\right), X_{s}\right\rangle d s+\frac{1}{t} \mathbb{E} \int_{0}^{t}\left\langle D \varphi\left(X_{s}\right), b X_{s}\right\rangle d s \\
\quad+\frac{1}{2 t} \mathbb{E} \int_{0}^{t} \operatorname{Tr}\left[D^{2} \varphi\left(X_{s}\right) Q\right] d s \\
\quad+\frac{1}{t} \mathbb{E} \int_{0}^{t} \int_{U}\left[\varphi\left(X_{s}+f\left(X_{s}, u\right)\right)-\varphi\left(X_{s}\right)\right. \\
\left.\quad-\left\langle D \varphi\left(X_{s}\right), f\left(X_{s}, u\right)\right\rangle\right] \lambda(d u) d s .
\end{aligned}
$$

Now, we shall prove that the expectation and the integral can be exchanged, $\mathbb{E}\left\langle A D \varphi\left(X_{s}\right), X_{s}\right\rangle, \mathbb{E}\left\langle D \varphi\left(X_{s}\right), b X_{s}\right\rangle, \mathbb{E} \operatorname{Tr}\left[D^{2} \varphi\left(X_{s}\right) Q\right]$ and $\mathbb{E}\left[\varphi\left(X_{s}+\right.\right.$ $\left.\left.f\left(X_{s}, u\right)\right)-\varphi\left(X_{s}\right)-\left\langle D \varphi\left(X_{s}\right), f\left(X_{s}, u\right)\right\rangle\right]$ are continuous with respect to $s$. 
Since $\left|i e^{i\left\langle X_{s}, h\right\rangle}\left\langle A h, X_{s}\right\rangle\right| \leq|A h|_{\mathbb{H}}\left|X_{s}\right|_{\mathbb{H}}$, Theorem 1.1 and Fubini's theorem imply that, for $0 \leq t \leq T$,

$$
\left|\frac{1}{t} \int_{0}^{t} \mathbb{E} i e^{i\left\langle X_{s}, h\right\rangle}\left\langle A h, X_{s}\right\rangle d s\right| \leq|A h|_{\mathbb{H}} \mathbb{E} \sup _{s \leq T}\left|X_{s}\right|_{\mathbb{H}} \leq C_{h, T}(1+V(x)) .
$$

By Theorem 1.1 and Proposition 2.1, one obtains

$$
\begin{aligned}
\mid \mathbb{E}\langle & \left.A D \varphi\left(X_{s}\right), X_{s}\right\rangle-\mathbb{E}\left\langle A D \varphi\left(X_{s^{\prime}}\right), X_{s^{\prime}}\right\rangle \mid \\
\leq & \mathbb{E}\left|e^{i\left\langle X_{s}, h\right\rangle}\left\langle A h, X_{s}\right\rangle-e^{i\left\langle X_{s^{\prime}}, h\right\rangle}\left\langle A h, X_{s}\right\rangle\right| \\
& +\mathbb{E}\left|e^{i\left\langle X_{s^{\prime}}, h\right\rangle}\left\langle A h, X_{s}\right\rangle-e^{i\left\langle X_{s^{\prime}}, h\right\rangle}\left\langle A h, X_{s^{\prime}}\right\rangle\right| \\
\leq & \left(\mathbb{E}\left|e^{i\left\langle X_{s}, h\right\rangle}-e^{i\left\langle X_{s^{\prime}}, h\right\rangle}\right|^{2}\right)^{1 / 2}\left(\mathbb{E}\left|\left\langle A h, X_{s}\right\rangle\right|^{2}\right)^{1 / 2}+\mathbb{E}\left|\left\langle A h, X_{s}-X_{s^{\prime}}\right\rangle\right| \\
= & {\left[\left.\mathbb{E}\left|\int_{0}^{1}\left\langle i h e^{i\left\langle\eta X_{s}+(1-\eta) X_{s^{\prime}}, h\right\rangle}, X_{s}-X_{s^{\prime}}\right\rangle d \eta\right|^{2}\right|^{1 / 2}\left(\mathbb{E}\left|\left\langle A h, X_{s}\right\rangle\right|^{2}\right)^{1 / 2}\right.} \\
& +|A h|_{\mathbb{H}} \mathbb{E}\left|X_{s}-X_{s^{\prime}}\right|_{\mathbb{H}} \\
\leq & |h|_{\mathbb{H}}|A h|_{\mathbb{H}}\left(\mathbb{E}\left|X_{s}-X_{s^{\prime}}\right|_{\mathbb{H}}^{2}\right)^{1 / 2}\left(\mathbb{E}\left|X_{s}\right|_{\mathbb{H}}^{2}\right)^{1 / 2} \\
& +|A h|_{\mathbb{H}} \mathbb{E}\left|X_{s}-X_{s^{\prime}}\right|_{\mathbb{H}} \rightarrow 0, \quad s \rightarrow s^{\prime} .
\end{aligned}
$$

We can similarly deal with the other terms on the right side of (5.1) as we just did in (5.2) for the first term. Thus we have

$$
\begin{aligned}
\lim _{t \rightarrow 0^{+}} & \frac{\mathbb{E} \varphi\left(X_{t}(x)\right)-\varphi(x)}{t} \\
= & \langle A D \varphi(x), x\rangle+\frac{1}{2} \operatorname{Tr}\left[D^{2} \varphi(x) Q\right]+\langle D \varphi(x), b(x)\rangle \\
& +\int_{U} \varphi(x+f(x, u))-\varphi(x)-\langle D \varphi(x), f(x, u)\rangle \lambda(d u),
\end{aligned}
$$

and $\sup _{t \in(0,1)}\left\|\mathbb{E} \varphi\left(X_{t}(x)\right)-\varphi(x)\right\|_{0, V} / t<\infty$.

(ii) The proof is similar to that of Lemma 3 in [18]. The term with $f$ can be dealt with similarly as the term of $b(\cdot)$. This completes the proof.

REMARK 5.1. Theorem 5.1 shows that we can easily obtain that $(\lambda I-$ $\left.\mathscr{K}_{0}\right)\left(\mathscr{E}_{A}(\mathbb{H})\right)$ is dense with respect to the $\pi$-convergence in $C_{b, V}\left(L^{6}\right)$. In fact, from Theorem 5.1, one has that $\mathscr{E}_{A}(\mathbb{H})$ is dense in $\mathscr{D}\left(\mathscr{K}, C_{b, V}\left(L^{6}\right)\right)$, this yields that it is dense in $C_{b, V}\left(L^{6}\right)$. Since $\lambda I-\mathscr{K}: \mathscr{D}\left(\mathscr{K}, C_{b, V}\left(L^{6}\right)\right) \rightarrow C_{b, V}\left(L^{6}\right)$ is bijective, we have that there exists $\psi \in \mathscr{D}\left(\mathscr{K}, C_{b, V}\left(L^{6}\right)\right)$ such that

$$
\varphi=(\lambda I-\mathscr{K}) \psi
$$

for any $\varphi \in C_{b, V}\left(L^{6}\right)$. And there exists $\left\{\psi_{n}\right\}_{n \in \mathbb{N}} \subset \mathscr{E}_{A}(\mathbb{H})$ such that

$$
\begin{gathered}
\frac{\psi_{n}}{1+V} \stackrel{\pi}{\rightarrow} \frac{\psi}{1+V}, \\
\frac{\mathscr{K}_{0} \psi_{n}}{1+V} \stackrel{\pi}{\rightarrow} \frac{\mathscr{K} \psi}{1+V} .
\end{gathered}
$$


These show

$$
\frac{\left(\lambda I-\mathscr{K}_{0}\right) \psi_{n}}{1+V} \stackrel{\pi}{\rightarrow} \frac{(\lambda I-\mathscr{K}) \psi}{1+V}=\varphi .
$$

By Theorem 5.1, we have that $P_{t}$ can act on $C_{b}\left(L^{6}(0,1)\right)$. Hence, the infinitesimal generator can be defined by

$$
\mathscr{K} \varphi(x)=\lim _{t \rightarrow 0^{+}} \frac{P_{t} \varphi(x)-\varphi(x)}{t}, \quad \varphi \in \mathscr{D}\left(\mathscr{K}, C_{b}\left(L^{6}(0,1)\right)\right), x \in L^{6}(0,1)
$$

with $\mathscr{D}\left(\mathscr{K}, C_{b}\left(L^{6}(0,1)\right)\right)=\left\{\varphi \in C_{b}\left(L^{6}(0,1)\right): \exists g \in C_{b}\left(L^{6}(0,1)\right)\right.$, $\lim _{t \rightarrow 0^{+}} P_{t} \varphi(x)-\varphi(x) / t=g(x), \forall x \in L^{6}(0,1)$, and $\sup _{t \in(0,1)}\left\|P_{t} \varphi-\varphi\right\|_{0} / t<$ $\infty\}$.

THEOREM 5.2. The family of linear maps $P_{t}^{*}:\left(C_{b}\left(L^{6}(0,1)\right)\right)^{*} \rightarrow\left(C_{b}\left(L^{6}(0\right.\right.$, $1)))^{*}, \quad t \geq 0$, defined by the formula, for $t \geq 0, F \in\left(C_{b}\left(L^{6}(0,1)\right)\right)^{*}, \varphi \in$ $C_{b}\left(L^{6}(0,1)\right)$,

$$
C_{b}\left(L^{6}(0,1)\right)\left\langle\varphi, P_{t}^{*} F\right\rangle_{\left(C_{b}\left(L^{6}(0,1)\right)\right)^{*}}=C_{b}\left(L^{6}(0,1)\right)\left\langle P_{t} \varphi, F\right\rangle_{\left(C_{b}\left(L^{6}(0,1)\right)\right)^{*}}
$$

is a linear operator semigroup of on $\left(C_{b}\left(L^{6}(0,1)\right)\right)^{*}$ which is stable on $\mathscr{M}\left(L^{6}(0,1)\right)$. Moreover, for any $\mu \in \mathscr{M}\left(L^{6}(0,1)\right)$ there exists an unique family of measures $\left\{\mu_{t}, t \geq 0\right\} \subset \mathscr{M}\left(L^{6}(0,1)\right)$ such that

$$
\begin{gathered}
\int_{0}^{T}\left|\mu_{t}\right|_{\mathrm{TV}}\left(L^{6}(0,1)\right) d t<\infty, \quad T>0 \\
\int_{L^{6}(0,1)} \varphi(x) \mu_{t}(d x)-\int_{L^{6}(0,1)} \varphi(x) \mu(d x)=\int_{0}^{t} \int_{L^{6}(0,1)} \mathscr{K} \varphi(x) \mu_{s}(d x) d s,
\end{gathered}
$$

for any $\varphi \in \mathscr{D}\left(\mathscr{K}, C_{b}\left(L^{6}(0,1)\right)\right), t \geq 0$. Finally, the solution of (5.5) satisfying (5.4) is given by $P_{t}^{*} \mu, t \geq 0$.

The proof is the same as the Theorem 6 in [18], so it is omitted.

By Theorem 3.2 and Theorem 3.3, we can extend Theorem 5.2 to the space $C_{b, V}\left(L^{6}\right)$.

THEOREM 5.3. Let $\left(P_{t}\right)_{t \geq 0}$ be the semigroup defined by equation (1.4) and the infinitesimal generator $\left(\mathscr{K}, \mathscr{D}\left(\mathscr{K}, C_{b, V}\left(L^{6}\right)\right)\right)$ is given by equation (1.5). Then, the formula

$$
\begin{aligned}
& \left\langle\varphi, P_{t}^{*} F\right\rangle_{\sigma\left(C_{b, V}\left(L^{6}(0,1)\right),\left(C_{b, V}\left(L^{6}(0,1)\right)\right)^{*}\right)} \\
& \quad=\left\langle P_{t} \varphi, F\right\rangle_{\sigma\left(C_{b, V}\left(L^{6}(0,1)\right),\left(C_{b, V}\left(L^{6}(0,1)\right)\right)^{*}\right)}
\end{aligned}
$$

defines a linear continuous operator semigroup $P_{t}^{*}$ on $\left(C_{b, V}\left(L^{6}(0,1)\right)\right)^{*}$, which is stable on $\mathscr{M}_{V}\left(L^{6}(0,1)\right)$. Moreover, for $\mu \in \mathscr{M}_{V}\left(L^{6}(0,1)\right)$ there exists an unique family of measures $\left\{\mu_{t}, t \geq 0\right\} \subset \mathscr{M}_{V}\left(L^{6}(0,1)\right)$ such that, for any $t \geq 0$, $\varphi \in \mathscr{D}\left(\mathscr{K}, C_{b, V}\left(L^{6}(0,1)\right)\right),(5.5)$ holds and

$$
\int_{0}^{T} \int_{L^{6}(0,1)}(1+V(x))\left|\mu_{t}\right|_{\mathrm{TV}}(d x) d t<\infty, \quad \forall T>0 .
$$


The solution of (5.5) satisfying (5.6) is given by $\left(P_{t}^{*} \mu\right)_{t \geq 0}$.

Proof. These are consequence of the essential propositions and theorems above. We give here a sketch of the proof. We mainly prove that the $P_{t}^{*} \mu$ is the unique solution of (5.5) satisfying (5.6).

Existence: Fixed $\mu \in \mathscr{M}_{V}\left(L^{6}(0,1)\right)$, we now show that for any $\varphi \in$ $\mathscr{D}\left(\mathscr{K}, C_{b, V}\left(L^{6}(0,1)\right)\right)$ the function

$$
\mathbb{R}_{+} \rightarrow \mathbb{R}, \quad s \mapsto \int_{L^{6}(0,1)} P_{s} \varphi(x) \mu(d x)
$$

is differentiable and the differential is given by

$$
s \mapsto \int_{L^{6}(0,1)} \mathscr{K} \varphi(x) P_{s}^{*} \mu(d x) .
$$

By (1.5) we have,

$$
\begin{aligned}
& \lim _{h \rightarrow 0^{+}} \frac{1}{h} \int_{L^{6}(0,1)}\left[P_{s+h} \varphi(x)-P_{s} \varphi(x)\right] \mu(d x) \\
&=\lim _{h \rightarrow 0^{+}} \int_{L^{6}(0,1)} \frac{P_{h} \varphi(x)-\varphi(x)}{h} P_{s}^{*} \mu(d x) \\
&=\int_{L^{6}(0,1)} \mathscr{K} \varphi(x) P_{s}^{*} \mu(d x) .
\end{aligned}
$$

Since $\mathscr{K} \varphi \in C_{b, V}\left(L^{6}(0,1)\right)$, we have

$$
\int_{L^{6}(0,1)} \mathscr{K} \varphi(x) P_{s}^{*} \mu(d x)=\int_{L^{6}(0,1)} P_{s} \mathscr{K} \varphi(x) \mu(d x) .
$$

Theorem 3.3 shows that this is a continuous function of $s$. By the fundamental theorem of calculus it follows that $\left(P_{t}^{*} \mu\right)_{t \geq 0}$ solves (5.5) satisfying (5.6).

Uniqueness: Assume that $\left\{\mu_{t}, t \geq 0\right\} \subset \mathscr{M}_{V}\left(L^{6}(0,1)\right)$ satisfies (5.5) and (5.6). It is straightforward to show that $\mathscr{D}\left(\mathscr{K}, C_{b}\left(L^{6}(0,1)\right)\right) \subset \mathscr{D}(\mathscr{K}$, $\left.C_{b, V}\left(L^{6}(0,1)\right)\right)$. Then $(5.5)$ holds for any $\varphi \in \mathscr{D}\left(\mathscr{K}, C_{b}\left(L^{6}(0,1)\right)\right)$. It is also obvious (5.6) implies (5.4). Then, Theorem 5.2 yields $\mu_{t}=P_{t}^{*} \mu$ for any $t \geq 0$.

THEOREM 5.4. For any $\mu \in \mathscr{M}_{V}\left(L^{6}(0,1)\right)$ there exists an unique family of measures $\left\{\mu_{t}, t \geq 0\right\} \subset \mathscr{M}_{V}\left(L^{6}(0,1)\right)$ satisfying (5.6) and the Fokker-Planck equation

$$
\int_{L^{6}(0,1)} \varphi(x) \mu_{t}(d x)-\int_{L^{6}(0,1)} \varphi(x) \mu(d x)=\int_{0}^{t} \int_{L^{6}(0,1)} \mathscr{K}_{0} \varphi(x) \mu_{s}(d x) d s
$$

for any $\varphi \in \mathscr{E}_{A}(\mathbb{H}), t \geq 0$. The solution is given by $P_{t}^{*} \mu, t \geq 0$, where $P_{t}^{*}:\left(C_{b, V}\left(L^{6}\right)\right)^{*} \rightarrow\left(C_{b, V}\left(L^{6}\right)\right)^{*}$ is the adjoint operator of $P_{t}, t \geq 0$. 
Proof. Existence: Fix $\mu \in \mathscr{M}_{V}\left(L^{6}(0,1)\right)$. Theorem 5.3 implies that $\left(P_{t}^{*} \mu\right)_{t \geq 0}$ satisfies (5.6). On the other hand, by Theorem 5.1 we have $\mathscr{E}_{A}(\mathbb{H}) \subset \mathscr{D}\left(\mathscr{K}, C_{b, V}\left(L^{6}(0,1)\right)\right)$ and $\mathscr{K} \varphi=\mathscr{K}_{0} \varphi$ for any $\varphi \in \mathscr{E}_{A}(\mathbb{H})$. It follows that (5.5) holds for any $t \geq 0, \varphi \in \mathscr{E}_{A}(\mathbb{H})$. Then, $P_{t}^{*} \mu, t \geq 0$ is solution of (5.7) satisfying (5.6).

Uniqueness: Assume that $\left\{\mu_{t}, t \geq 0\right\} \subset \mathscr{M}_{V}\left(L^{6}(0,1)\right)$ fulfilling (5.7) with (5.6). For $\varphi \in C_{b, V}\left(L^{6}\right)$, Theorem 5.1 yields that there exist $m \in \mathbb{N}$ and an $m$-indexed sequence $\left\{\varphi_{n_{1}, \ldots, n_{m}}\right\}_{\left(n_{1}, \ldots, n_{m}\right) \in \mathbb{N}^{m}} \subset \mathscr{E}_{A}(\mathbb{H})$ such that

$$
\begin{array}{r}
\lim _{n_{1} \rightarrow \infty} \cdots \lim _{n_{m} \rightarrow \infty} \frac{\varphi_{n_{1}, \ldots, n_{m}}}{1+V} \stackrel{\pi}{=} \frac{\varphi}{1+V}, \\
\lim _{n_{1} \rightarrow \infty} \cdots \lim _{n_{m} \rightarrow \infty} \frac{\mathscr{K}_{0} \varphi_{n_{1}, \ldots, n_{m}}}{1+V} \stackrel{\pi}{=} \frac{\mathscr{K} \varphi}{1+V} .
\end{array}
$$

Since $\left\{\mu, \mu_{t}, t \geq 0\right\} \subset \mathscr{M}_{V}\left(L^{6}(0,1)\right)$, the dominated convergence theorem shows

$$
\begin{aligned}
& \lim _{n_{1} \rightarrow \infty} \ldots \lim _{n_{m} \rightarrow \infty}\left(\int_{L^{6}(0,1)} \varphi_{n_{1}, \ldots, n_{m}}(x) \mu_{t}(d x)-\int_{L^{6}(0,1)} \varphi_{n_{1}, \ldots, n_{m}}(x) \mu(d x)\right) \\
& =\int_{L^{6}(0,1)} \varphi(x) \mu_{t}(d x)-\int_{L^{6}(0,1)} \varphi(x) \mu(d x)
\end{aligned}
$$

and for any $s \in[0, t]$,

$$
\lim _{n_{1} \rightarrow \infty} \cdots \lim _{n_{m} \rightarrow \infty} \int_{L^{6}(0,1)} \mathscr{K} \varphi_{n_{1}, \ldots, n_{m}}(x) \mu_{s}(d x)=\int_{L^{6}(0,1)} \mathscr{K} \varphi(x) \mu_{s}(d x) .
$$

By using (5.6) and the dominated convergence theorem, we have

$$
\begin{aligned}
& \lim _{n_{1} \rightarrow \infty} \ldots \lim _{n_{m} \rightarrow \infty} \int_{0}^{t}\left(\int_{L^{6}(0,1)} \mathscr{K}_{0} \varphi_{n_{1}, \ldots, n_{m}}(x) \mu_{s}(d x)\right) d s \\
& =\int_{0}^{t}\left(\int_{L^{6}(0,1)} \mathscr{K} \varphi(x) \mu_{s}(d x)\right) d s .
\end{aligned}
$$

This means that $\left\{\mu_{t}, t \geq 0\right\}$ is the solution of (5.5) satisfying (5.6). Theorem 5.3 shows the uniqueness of the solution. Hence, $\mu_{t}$ must coincide with $P_{t}^{*} \mu, t \geq 0$.

Acknowledgment. The authors are very grateful to Professor Jianglun Wu for his detailed comments and criticisms which helped to produce a clearer exposition and the elimination of several inaccuracies from the first manuscript.

\section{REFERENCES}

[1] M. Balázs, J. Quastel and T. Seppäläinen, Fluctuation exponent of the KPZ/stochastic Burgers equation, J. Amer. Math. Soc. 24 (2011), 683-708. MR 2784327

[2] J. B. Bell, J. Foo and A. L. Garcia, Algorithm refinement for the stochastic Burgers' equation, J. Comput. Phys. 223 (2007), 451-468. MR 2314398 
[3] L. Bertini, N. Cancrini and G. Jona-Lasinio, The stochastic Burgers equation, Comm. Math. Phys. 165 (1994), 211-232. MR 1301846

[4] G. Da Prato and A. Debussche, $m$-dissipativity of Kolmogorov operators corresponding to Burgers equations with space-time white noise, Potential Anal. 26 (2007), 31-35. MR 2276524

[5] G. Da Prato, A. Debussche and R. Temam, Stochastic Burgers' equation, NoDEA Nonlinear Differential Equations Appl. 1 (1994), 389-402. MR 1300149

[6] G. Da Prato and J. Zabczyk, Stochastic equations in infinite dimensions, Cambridge University Press, Cambridge, 1992. MR 1207136

[7] G. Da Prato and J. Zabczyk, Ergodicity for infinite-dimensional systems, Cambridge University Press, Cambridge, 1996. MR 1417491

[8] Z. Dong and Y. C. Xie, Global solutions of stochastic 2D Navier-Stokes equations with Lévy noise, Sci. China Ser. A 52 (2009), 1497-1524. MR 2520590

[9] Z. Dong and Y. C. Xie, Ergodicity of linear SPDE driven by Lévy noise, J. Syst. Sci. Complex. 23 (2010), 137-152. MR 2595875

[10] Z. Dong and Y. C. Xie, Ergodicity of stochastic 2D Navier-Stokes equation with Lévy noise, J. Differential Equations 251 (2011), 196-222. MR 2793269

[11] Z. Dong and T. G. Xu, One-dimensional stochastic Burgers equation driven by Lévy processes, J. Funct. Anal. 243 (2007), 631-678. MR 2289699

[12] F. Flandoli, Dissipativity and invariant measures for stochastic Navier-Stokes equation, NoDEA Nonlinear Differential Equations Appl. 1 (1994), 403-423. MR 1300150

[13] C. Giraud, Genealogy of shocks in Burgers turbulence with white noise initial velocity, Comm. Math. Phys. 223 (2001), 67-86. MR 1860760

[14] M. Gourcy, Large deviation principle of occupation measure for stochastic Burgers equation, Ann. Inst. Henri Poincaré Probab. Stat. 43 (2007), 441-459. MR 2329511

[15] E. Hopf, The partial differential equation $u_{t}+u u_{x}=\mu u_{x x}$, Comm. Pure Appl. Math. 3 (1950), 201-230. MR 0047234

[16] W. E, K. Khanin, A. Mazel and Y. G. Sinai, Invariant measures for Burgers equation with stochastic forcing, Ann. of Math. (2) 151 (2000), 877-960. MR 1779561

[17] Y. Liu and H. Z. Zhao, Representation of pathwise stationary solutions of stochastic Burgers' equations, Stoch. Dyn. 9 (2009), 613-634. MR 2589039

[18] L. Manca, The Kolmogorov operator associated to a Burgers SPDE in spaces of continuous functions, Potential Anal. 32 (2010), 67-99. MR 2575387

[19] A. D. Neate and A. Truman, A one-dimensional analysis of real and complex turbulence and the Maxwell set for the stochastic Burgers equation, J. Phys. A 38 (2005), 7093-7127. MR 2167533

[20] E. Priola, On a class of Markov type semigroups in spaces of uniformly continuous and bounded functions, Studia Math. 136 (1999), 271-295. MR 1724248

[21] Y. G. Sinai, Statistics of shocks in solution of inviscid Burgers equation, Comm. Math. Phys. 148 (1992), 601-621. MR 1181071

[22] A. Truman and J. L. Wu, Stochastic Burgers equation with Lévy space-time white noise, Probabilistic methods in fluids, Proceedings of the Swansea 2002 Workshop, World Sci. Publishing, River Edge, NJ, 2003, pp. 298-323. MR 2083380

[23] A. Truman and J. L. Wu, On a stochastic nonlinear equation arising from $1 D$ integro-differential scalar conservation laws, J. Funct. Anal. 238 (2006), 612-635. MR 2253735

[24] Y. C. Xie, Poincaré inequality for linear SPDE driven by Lévy noise, Stochastic Process. Appl. 120 (2010), 1950-1965. MR 2673983

Bing Hu, School of Mathematics and Statistics, Jiangsu Normal University, Xuzhou 221116, China 
Xiaobin Sun, School of Mathematics and Statistics, Jiangsu Normal University, Xuzhou 221116, China

Yingchao Xie, School of Mathematics and Statistics, Jiangsu Normal UniverSITY, XUZHOU 221116, ChinA

E-mail address: ycxie@jsnu.edu.cn 